ARTICLE

\title{
Colonization of dermal arterioles by Neisseria meningitidis provides a safe haven from neutrophils
}

\author{
Valeria Manriquez ${ }^{1}$, Pierre Nivoit ${ }^{1}$, Tomas Urbina', Hebert Echenique-Rivera', Keira Melican (1) 1,5, \\ Marie-Paule Fernandez-Gerlinger ${ }^{1}$, Patricia Flamant ${ }^{2}$, Taliah Schmitt ${ }^{3}$, Patrick Bruneval ${ }^{4}$, Dorian Obino ${ }^{1 凶} \&$ \\ Guillaume Duménil (D) ${ }^{1 \times}$
}

The human pathogen Neisseria meningitidis can cause meningitis and fatal systemic disease. The bacteria colonize blood vessels and rapidly cause vascular damage, despite a neutrophilrich inflammatory infiltrate. Here, we use a humanized mouse model to show that vascular colonization leads to the recruitment of neutrophils, which partially reduce bacterial burden and vascular damage. This partial effect is due to the ability of bacteria to colonize capillaries, venules and arterioles, as observed in human samples. In venules, potent neutrophil recruitment allows efficient bacterial phagocytosis. In contrast, in infected capillaries and arterioles, adhesion molecules such as E-Selectin are not expressed on the endothelium, and intravascular neutrophil recruitment is minimal. Our results indicate that the colonization of capillaries and arterioles by $N$. meningitidis creates an intravascular niche that precludes the action of neutrophils, resulting in immune escape and progression of the infection.

\footnotetext{
${ }^{1}$ Pathogenesis of Vascular Infections unit, INSERM, Institut Pasteur, Paris, France. ${ }^{2}$ Experimental Neuropathology Unit, Institut Pasteur, Paris, France. ${ }^{3}$ Paris Saint-Joseph Hospital, Paris, France. ${ }^{4}$ Service d'Anatomie Pathologie, Hôpital Européen Georges Pompidou, Assistance Publique-Hôpitaux de Paris (AP-HP), Paris, France. ${ }^{5}$ Present address: Department of Neuroscience, Karolinska Institutet, Stockholm, Sweden. ${ }^{\varpi_{e}}$ ail: dorian.obino@pasteur.fr; guillaume. dumenil@pasteur.fr
} 
nfections caused by Neisseria meningitidis are characterized by an unusually fast progression of the disease as if bacterial proliferation could not be contained by the innate immune system. Although N. meningitidis is best known for causing cerebrospinal meningitis, the systemic septic form of the infection is responsible for $90 \%$ of the mortality attributed to meningococcal infections ${ }^{1,2}$. In both cases, the infection is characterized by a fulminant evolution and diseases can progress from relatively unspecific flu-like symptoms to a life-threatening condition in less than $24 \mathrm{~h}^{1}$. Meningococcal systemic infections are characterized by a typical skin rash, called purpura, indicating a perturbation of the vascular function ${ }^{3,4}$. Clinical studies have revealed major perturbations in vascular function in infected tissues with congestion, coagulation, and the loss of vascular integrity ${ }^{5,6}$. Neisseria meningitidis thus rapidly causes major vascular damages leading to a fatal outcome in absence of treatment as if the first lines of immune defense were not sufficient.

How $N$. meningitidis evades innate immunity remains largely unknown. More specifically, an intriguing question that remains is how this bacterium escapes the neutrophil response triggered by the infection. Indeed, both clinical and experimental results point to the recruitment of an inflammatory cellular infiltrate ${ }^{5,7}$ including neutrophils, macrophages, and monocytes, which are typically seen around infected blood vessels ${ }^{5-7}$. However, although both neutrophils and monocytic cells frequently contain bacteria $^{5,7}$, this is not sufficient to clear the infection. The recruitment of neutrophils to infected tissues was also observed in a recently developed humanized mouse model in which grafting human skin allows the formation of a network of human dermal vessels that anastomose with the murine dermal vasculature and are perfused with murine blood ${ }^{8}$. In this model, intravenous bacteria exclusively adhere to the human endothelium, progressively proliferate to occupy the entire vessel lumen and recapitulate the human disease. Taken together, these studies illustrate the ability of the host to initiate an innate immune response upon meningococcal infections but raise pivotal questions regarding why neutrophils fail to clear these infections.

In this study, we hypothesized that the immune escape abilities of Neisseria meningitidis are linked to its ability to occupy an intravascular niche. This feature is not unique to $N$. meningitidis but extends to a number of bacterial and viral pathogens that can reach the blood circulation, interact with the endothelium and colonize vessels, including SARS-COV2, as recently described ${ }^{9-11}$. Of note, intravascular colonization by these pathogens generates an atypical situation in terms of neutrophil recruitment. Indeed, the textbook description in which bacteria are found in tissues and neutrophils are recruited to that sites following their rolling on the endothelium of postcapillary venules followed by their extravasation cannot apply for pathogens residing inside blood vessels. Whether the intravascular localization of Neisseria meningitidis might account for the incapacity of the innate immune system to clear this infection remains an open question. Therefore, studying the interaction of intravascular bacterial and viral pathogens with endothelial cells is critical to better understand their pathogenesis.

In this work, using a humanized mouse model of meningococcal infection and human samples, we show that Neisseria meningitidis escapes the neutrophil response by infecting arterioles and capillaries. In these vessel types, neutrophils are not recruited despite intense intravascular infection. $N$. meningitidis thus occupies a specific niche in the vasculature that protects them from the neutrophil response.

\section{Results}

Dynamics of neutrophil recruitment to sites of infection. As previously shown ${ }^{8,12}$, Neisseria meningitidis specifically colonize human vessels within a few hours (Fig. 1a and Supplementary Fig. 1a, b). Intravital time-lapse imaging shows bacteria adhering to the vascular wall (Supplementary Movie 1), proliferating in the form of aggregates that become apparent after $2 \mathrm{~h}$ and progressively fuse to finally occupy the vascular space at $6 \mathrm{~h}$ post infection (p.i.). The absence of colonization of mouse vessels confirms the sharp species specificity of the interaction with the endothelium. Efficient heterotypic interactions between murine neutrophils and the human endothelium in our model were confirmed by subcutaneous injection of human TNFa (Supplementary Movie 2), as previously described ${ }^{13}$.

Neutrophil recruitment to infected vessels was assessed by immunofluorescence of histological samples, flow cytometry, and intravital imaging. Immunohistological staining of infected skin tissues revealed that only a few neutrophils could be found within the infected tissue $3 \mathrm{~h}$ p.i., with numbers of neutrophils accumulating in the vicinity of infected vessels progressively increasing at $6 \mathrm{~h}$ p.i. onward (Fig. 1b). Quantitative analysis by flow cytometry of dissociated tissue revealed that neutrophils continued to accumulate up to $24 \mathrm{~h}$ p.i., whereas contralateral mouse skin tissues did not show this neutrophil accumulation (Fig. 1c and Supplementary Fig. 1c). Intravital imaging of 56 infected vessels confirmed the recruitment of neutrophils during the early phase of the infection $(0-6 \mathrm{~h})$ but only $46 \%$ of the infected vessels exhibited at least one neutrophil in their proximity $6 \mathrm{~h}$ p.i., pointing to a heterogeneous neutrophil response across the infected tissue (Supplementary Fig. 1d). Similarly, the numbers of neutrophils per $\mathrm{mm}^{2}$ of endothelium progressively increased and reached the mean value of $93 \pm 27$ neutrophils $6 \mathrm{~h}$ p.i. (Fig. 1d). The secretion of murine cytokines in infected tissues was in good agreement with the kinetics of neutrophil recruitment. Murine neutrophil-attracting cytokines such as TNF, CXCL1, and CXCL2 were detectable as early as $6 \mathrm{~h}$ p.i. and progressively increased with time (Supplementary Fig. 2a). Similarly, human neutrophil-recruiting cytokines such as TNF and IL- 8 were also produced by the human endothelium upon infection (Supplementary Fig. 2b).

Reminiscent of human cases ${ }^{7}$, at $16 \mathrm{~h}$ p.i., the recruitment of LysM ${ }^{\mathrm{GFP}}$-labeled neutrophils visualized by intravital imaging was massive with certain infected vessels being surrounded by large numbers of neutrophils, forming a sheath around the vessel (Fig. 1e and Supplementary Movie 3). Monocytes also express the LysM $^{\mathrm{GFP}}$ reporter but were easily distinguished from neutrophils by their low level of fluorescence ${ }^{14}$. Single-cell tracking showed that neutrophils converge from the parenchyma toward the infected vessel (Fig. 1e and Supplementary Movie 3). Together these observations demonstrate that neutrophils progressively accumulate in the vicinity of certain infected vessels following $N$. meningitidis vascular colonization, starting at $3 \mathrm{~h}$ post infection and reaching large amounts at $16-24 \mathrm{~h}$ p.i., as observed in human cases.

Intravascular colonization determines the nature and timing of neutrophil recruitment. We next investigated the bacterial signals involved in neutrophil recruitment. To determine whether neutrophil recruitment relied on vascular colonization by $N$. meningitidis, we studied mutants that have altered ability to interact along the vascular wall. Grafted mice were first infected with two isogenic bacterial strains, pilC1, a piliated but nonadherent mutant, and pilD, a mutant that does not express any pili on its surface ${ }^{15}$ (Fig. 2a). Both strains cannot adhere to the endothelium and thus cannot colonize vessels. As expected ${ }^{8}$, while mice were inoculated with similar amounts of bacteria (Fig. 2b), the numbers of adherent bacteria $24 \mathrm{~h}$ p.i. were strongly decreased when mice were infected with the mutant bacterial 
a

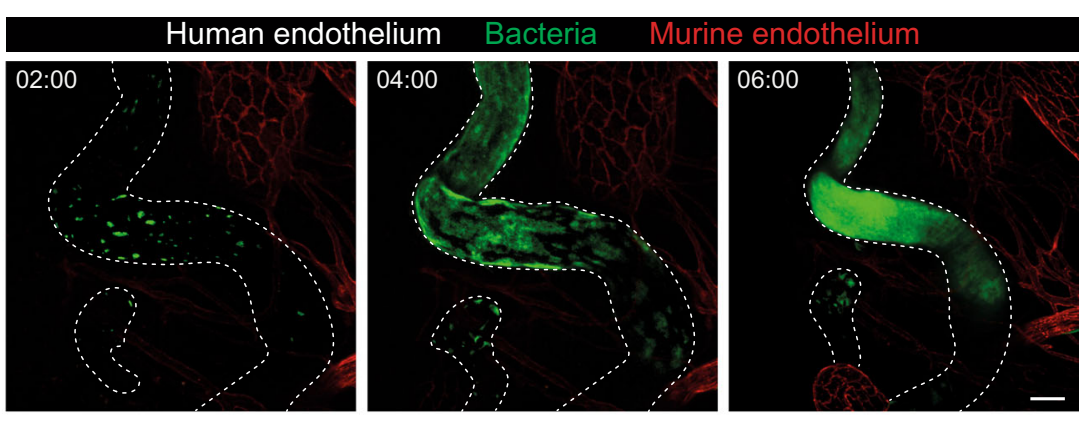

b
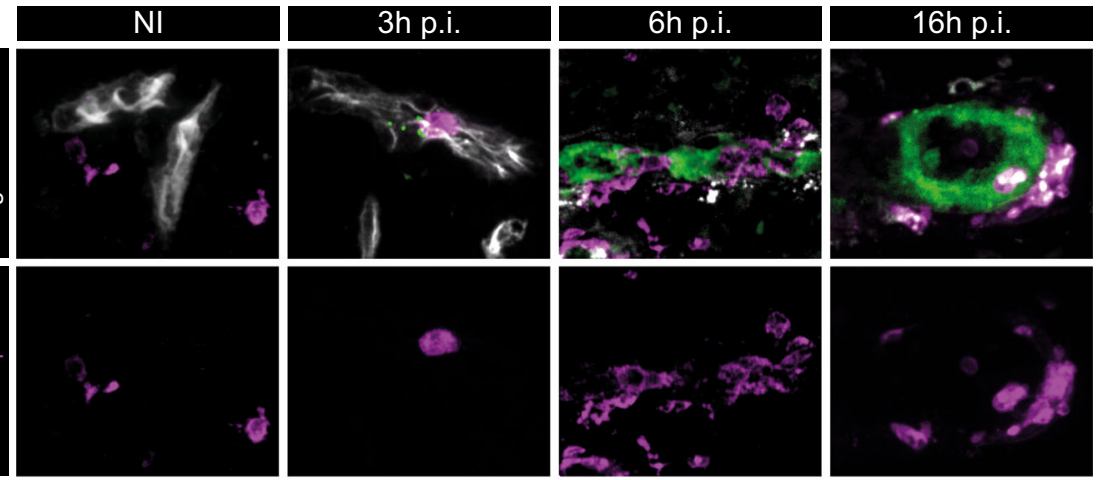
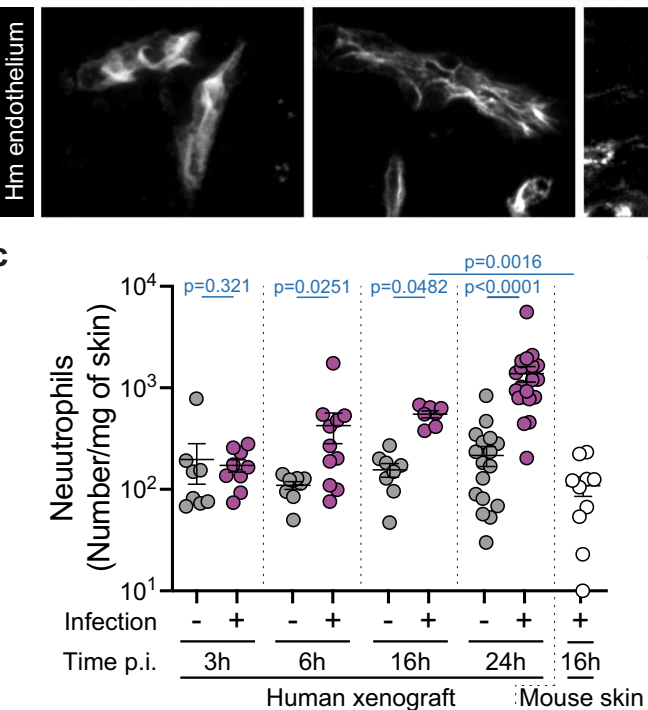
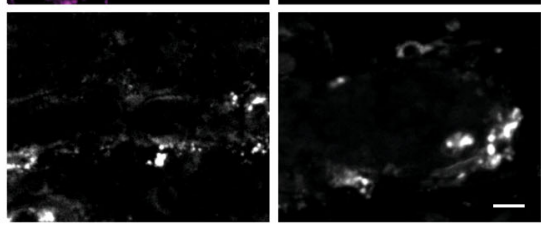

d

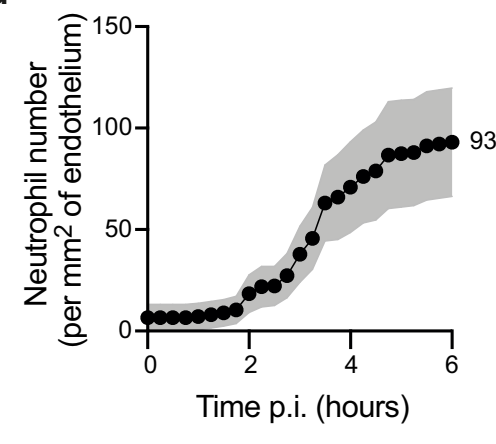

e

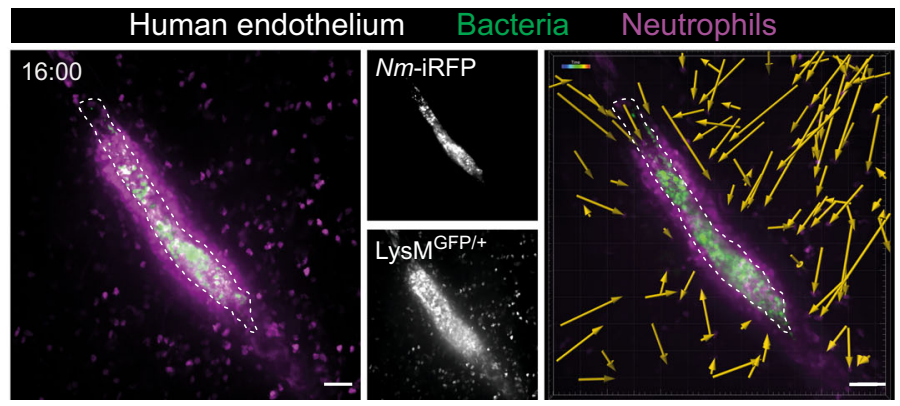

strains (Fig. 2c). We found that neutrophil recruitment $24 \mathrm{~h}$ after infection was tightly dependent on bacterial adhesion since their numbers barely increased when mice were infected with either of the mutant bacterial strains (Fig. 2d).

Type IV pili also allow bacteria to form three-dimensional viscous aggregates containing thousands of bacteria and participating in vessel occlusion ${ }^{12}$. To test the impact of these bacterial aggregates inside blood vessels on neutrophil recruitment, a strain expressing a sequence variant (SA) of the major pilin unable to generate auto-aggregation while still adhering to endothelial cells ${ }^{16}$ was compared to the wild-type sequence variant (SB) (Fig. 2e). While both strains showed the same ability to survive inside the circulation, the SA variant showed lower amounts of bacteria colonizing blood vessels as expected (Fig. 2f, g). The 
Fig. 1 Recruitment of neutrophils to sites of infection. a Representative time sequence (maximum intensity z-projection) of vascular colonization by iRFPexpressing Neisseria meningitidis (green). The human and murine endothelia were distinguished using UEA-1 lectin (dashed lines) and mouse-specific antiCD31 (red) staining, respectively. Time, hh:min. Pictures shown are representatives of $N=6$ mice. Scale bar, $50 \mu$ m. b Representative images (maximum intensity z-projection) of non-infected (NI) and infected human graft tissue sections at indicated times post infection (p.i.) with GFP-expressing Neisseria meningitidis (green) and stained for the human endothelium (UEA-1 lectin, gray) and neutrophils (GR-1, magenta). Pictures shown are representative of $N=3$ mice per condition. Scale bar, $10 \mu \mathrm{m}$. c Quantification of neutrophil numbers in human xenografts of non-infected (-, gray circles) and infected $(+$, purple circles) mice at the indicated times post infection (p.i.). Neutrophil numbers in contralateral mouse skin at $16 \mathrm{~h}$ post infection is shown on the right (white circles). Two-tailed Mann-Whitney test ( $3 \mathrm{~h}, 6 \mathrm{~h}, 24 \mathrm{~h})$ and two-tailed Kruskal-Wallis test with Dunn's correction ( $16 \mathrm{~h}$ ). $n=7$ ( $16 \mathrm{~h} \mathrm{p.i.),} 8$ ( 3 h, 16 h non-infected; 6 h p.i.), 9 (3 h p.i., 6 h non-infected), 10 (mouse skin, 16 h p.i.), 18 ( 24 h non-infected), and 21 ( 24 h p.i.) mice, pooled from $N=3$ ( 3 h, $6 \mathrm{~h}, 16 \mathrm{~h}$ p.i.) and 5 ( $24 \mathrm{~h}$ p.i.) independent experiments. $\mathbf{d}$ Movies obtained from intravital imaging were used to quantify the numbers of neutrophils per square millimeter of endothelium during the first $6 \mathrm{~h}$ of the infection. Quantifications were performed on $n=56$ vessels, pooled from $N=7$ infected mice imaged independently. c, d Data are shown as the mean \pm SEM. e Intravital imaging (maximum intensity z-projection) of neutrophil (LysM ${ }^{G F P /+}$, magenta) recruitment at $16 \mathrm{~h}$ post infection with iRFP-expressing Neisseria meningitidis (green) in grafted $\mathrm{Rag}_{2}{ }^{-/-} \gamma_{\mathrm{c}}{ }^{-/-}$LysMGFP/+ mice. Dashed lines delineate the human endothelium, stained using the UEA-1 lectin. The left panel shows an important swarm of neutrophils around the infected vessel. Time, hh:min. Scale bars, $50 \mu \mathrm{m}$. The right panel shows the directed migration of neutrophils towards the infected vessel as revealed by cell tracking over 30 min. Pictures shown are representative of $N=3$ mice. Scale bar, $50 \mu \mathrm{m}$.

a

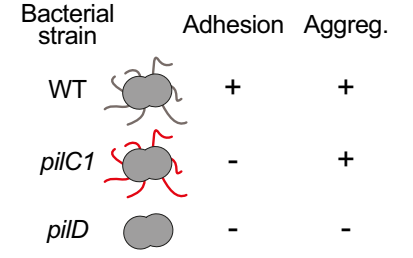

e

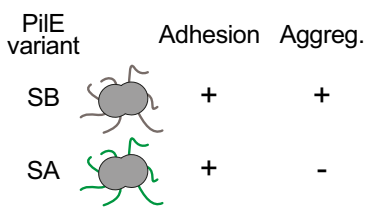

b

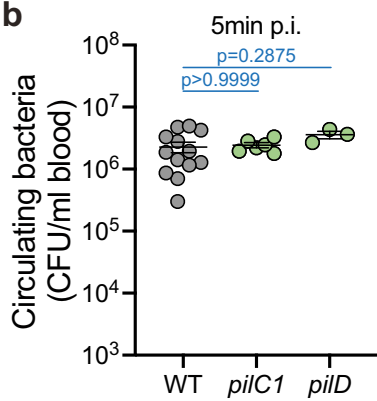

f

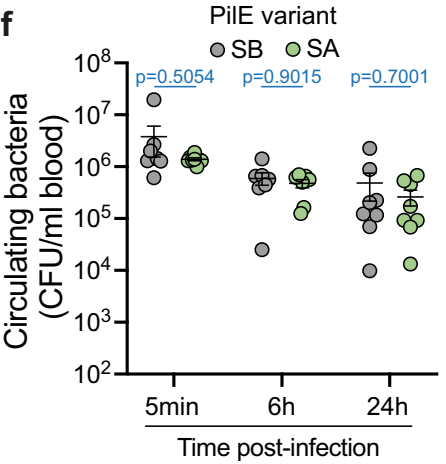

C

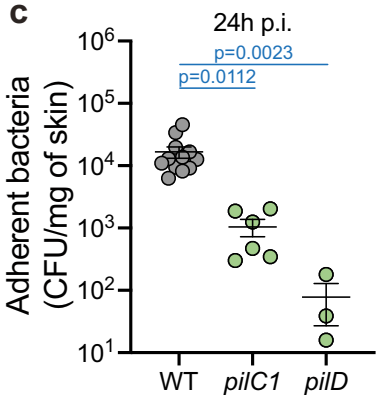

g

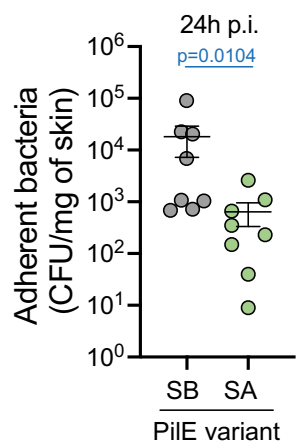

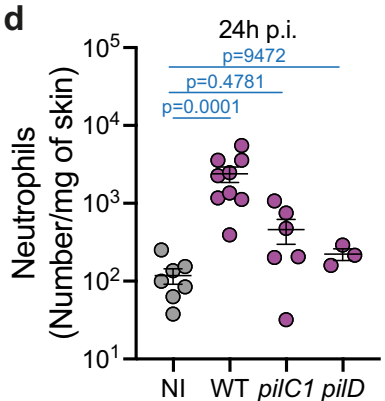

h

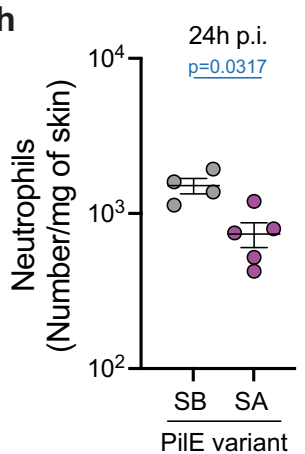

Fig. 2 Neutrophil recruitment to infected vessels relies on type IV pili-mediated bacterial adhesion and aggregation. a Schematic representation of the bacterial strains used to assess the impact of bacterial adhesion on neutrophil recruitment. $\mathbf{b}$ Bacterial colony-forming unit (CFU) counts from blood of mice infected for $5 \mathrm{~min}$ with the indicated bacterial strains. Two-tailed Kruskal-Wallis test with Dunn's correction. $n=13,6,3$ mice for WT, pilC1, pilD, respectively, pooled from $N=3$ independent experiments (except for pilD $N=1$ ). c Bacterial CFU counts from dissociated xenografts collected from mice infected for $24 \mathrm{~h}$ with the indicated bacterial strains. Two-tailed Kruskal-Wallis test with Dunn's correction. $n=12,6,3$ mice for WT, pilC1, pilD, respectively, pooled from $N=3$ independent experiments (except for pilD $N=1$ ). d Quantification of neutrophil numbers in human xenografts harvested from non-infected $(\mathrm{NI})$ mice and mice infected for $24 \mathrm{~h}$ with the indicated bacterial strains. Two-tailed Kruskal-Wallis test with Dunn's correction. $n=7,9$, 9, 3 mice for non-infected, WT, pilC1, pilD, respectively, pooled from $N=3$ independent experiments (except for pilD $N=1$ ). b-d Data are shown as the mean \pm SEM. e Schematic representation of the bacterial strains used to assess the impact of bacterial aggregation on neutrophil recruitment. $\mathbf{f}$ Bacterial CFU counts from blood of mice infected for the indicated times with SB (gray circles) or SA (green circles) pilin variant-expressing bacteria. Two-tailed Mann-Whitney test per time point. $n=8$ mice per bacterial strain ( 5 min and $24 \mathrm{~h}$ p.i.) and 7 mice per bacterial strain $(6 \mathrm{~h}$ p.i.), pooled from $N=3$ independent experiments. $\mathbf{g}$ Bacterial CFU counts from dissociated xenografts collected from mice infected for $24 \mathrm{~h}$ with SB or SA pilin variant-expressing bacteria. Two-tailed Mann-Whitney test. $n=8$ (SB) and 9 (SA) mice, pooled from $N=3$ independent experiments. $\mathbf{h}$ Quantification of neutrophil numbers in xenografts harvested from mice infected for $24 \mathrm{~h}$ with SB or SA pilin variant-expressing bacteria. Two-tailed Mann-Whitney test. $n=4$ (SB) and 5 mice $(\mathrm{SA})$, pooled from $N=2$ independent experiments. $\mathbf{f}-\mathbf{h}$ Data are shown as the mean $\pm \mathrm{SEM}$.

amounts of neutrophils recruited by the non-aggregative strain were reduced (Fig. 2h), showing that the formation of bacterial aggregates enhanced neutrophil recruitment. These data show that bacterial adhesion, amplified by bacterial auto-aggregation, both mediated by type IV pili, is essential to initiate a cascade eventually leading to neutrophil recruitment.
Neutrophils only partially control the number of colonizing bacteria and subsequent vessel damages. To address the role of recruited neutrophils during meningococcal disease, we depleted these cells and evaluated the effect on infection. Grafted mice were pre-treated with the monocytes and neutrophil-depleting antibody directed against GR-1 (clone RB6-8C5), $24 \mathrm{~h}$ prior to 


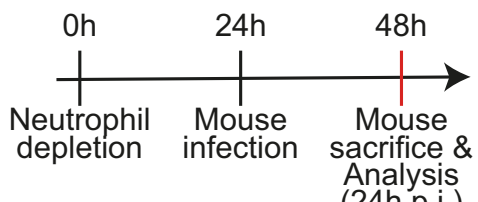

(24h p.i.)

C

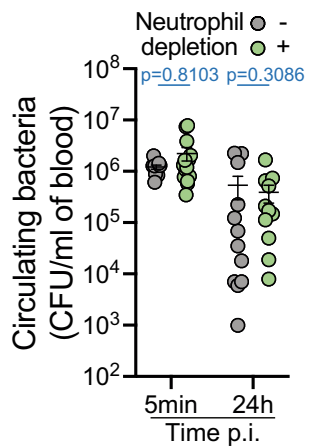

f

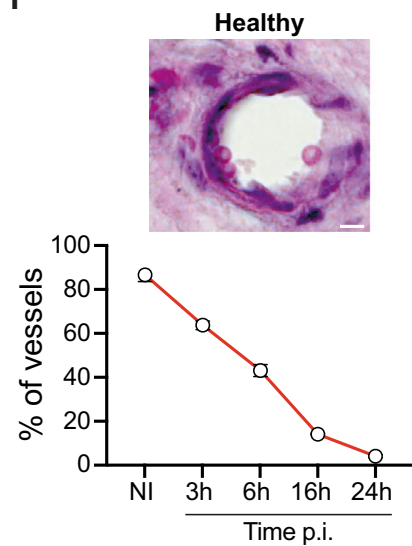

g

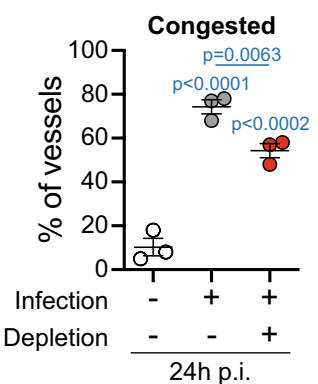

d
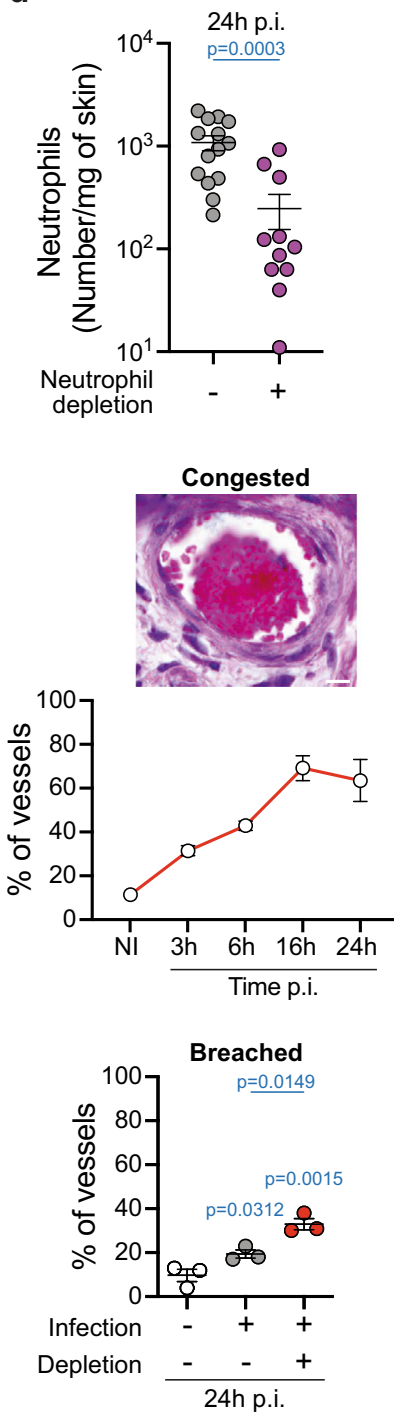

b

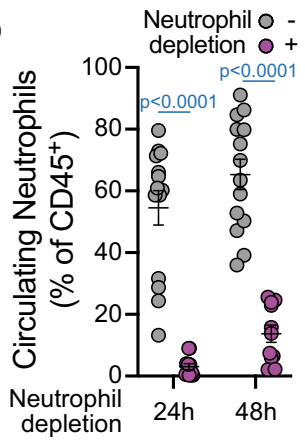

e

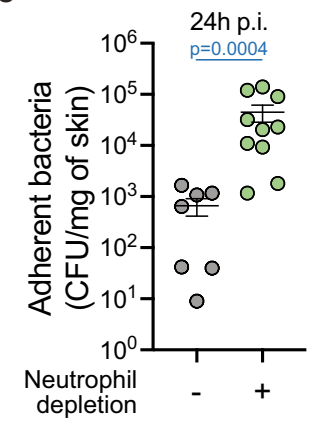

h

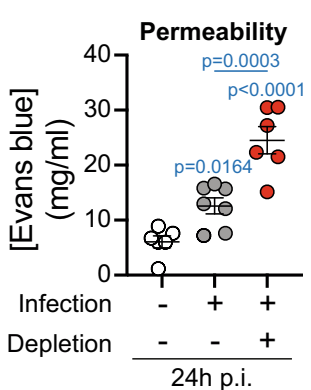

infection (Fig. 3a). This treatment led to efficient depletion of circulating neutrophils when compared to the mice that received the isotype control antibody (Fig. 3b). The absence of neutrophils in the circulation did not affect the number of nonadherent circulating bacteria after intravenous injection at any of the tested times post infection (Fig. 3c). As expected, the depletion effectively prevented the neutrophil recruitment to infected human tissues (Fig. 3d). Importantly, the number of adherent bacteria at $24 \mathrm{~h}$ p.i. was strongly increased when compared to the control condition (Fig. 3e). Nearly identical results were obtained when the mice were treated with the neutrophil-specific depletion antibody clone 1A8, against Ly-6G, supporting that monocytes likely do not further contribute to bacterial clearance (Supplementary Fig. 3a-d). These results indicate that neutrophils are the primary cell type controlling the number of bacteria adhering and proliferating along the endothelium, but are unable to completely resolve the infection within this time frame.

As pathological effects of $\mathrm{Nm}$ infections are linked to altered vascular function, we also measured the potential contribution of neutrophils to vascular damage. Indeed, neutrophil has been reported to induce vascular damage in other systems ${ }^{17}$, and infection by Neisseria meningitidis in humans has been shown to involve vascular damage ${ }^{7,8}$. Kinetics of vascular damage after $N$. meningitidis infection were first determined using histology and 
Fig. 3 Neutrophils limit vascular colonization by meningococci and vessel damages. a Schematic of the approach used to deplete neutrophils using the neutrophil-depleting antibody anti-GR-1, clone RB6-8C5, and infect mice. b Numbers of blood circulating neutrophils in non-infected mice pre-treated with the isotype control (-, gray circles) or the neutrophil-depleting ( + , purple circles) antibody. Two-tailed Mann-Whitney test per time point. $n=14$ (control) and 11 (depletion) mice per time point, pooled from $N=4$ independent experiments. c Bacterial CFU counts from blood of mice pre-treated with the isotype control (-, gray circles) or the neutrophil-depleting ( + , green circles) antibody and infected for the indicated time points. Two-tailed Mann-Whitney test per time point. $n=12$ (control, $5 \mathrm{~min}$ and $24 \mathrm{~h}$ p.i.), 14 and 11 (depletion, $5 \mathrm{~min}$ and $24 \mathrm{~h}$ p.i., respectively) mice, pooled from $N=4$ independent experiments. $\mathbf{d}$ Neutrophil numbers in the xenografts of mice pre-treated with the isotype control (-, gray circles) or the neutrophil-depleting ( + , purple circles) antibody and infected for $24 \mathrm{~h}$. Two-tailed Mann-Whitney test per time point. $n=14$ (control) and 12 (depletion) mice, pooled from $N=4$ independent experiments. e Bacterial CFU counts from dissociated xenografts collected from mice pre-treated with the isotype control (-, gray circles) or the neutrophil-depleting ( + , green circles) antibody and infected for $24 \mathrm{~h}$. Two-tailed Mann-Whitney test. $n=7$ (control) and 10 (depletion) mice, pooled from $N=3$ independent experiments. $\mathbf{f}$ Representative images of hematoxylin and eosin-stained histological sections of infected xenografts and quantification of vascular damage in infected vessels. Quantifications were performed on $n=500$ vessels, pooled from $N=5$ (non-infected), 4 ( $3 \mathrm{~h}$ p.i.) and 3 ( $6 \mathrm{~h}, 16 \mathrm{~h}$, and $24 \mathrm{~h}$ p.i.) mice. Scale bar, $10 \mu \mathrm{m}$. $\mathbf{g}$ Quantification of vascular damage upon mouse infection and neutrophil depletion. Two-tailed oneway ANOVA with Holm-Sidak's correction. Quantifications were performed on $n=200$ vessels, pooled from $N=3$ mice per group. $\mathbf{h}$ Quantification of vascular permeability upon mouse infection and neutrophil depletion with the anti-Ly-6G antibody (clone 1A8) using Evans blue. Two-tailed one-way ANOVA with Holm-Sidak's correction. $n=6$ (non-infected and Infected with neutrophil depletion) and 7 (infected without neutrophil depletion) mice, pooled from $N=4$ independent experiments. b-h Data are shown as the mean $\pm \mathrm{SEM}$.

intravital imaging to compare with neutrophil recruitment kinetics. Over 500 blood vessels on histological slices were analyzed for each time point and classified as healthy, congested (with accumulation of red blood cells), or breached (with perivascular extravasation of red blood cells). Representative images and quantitative results of each vascular condition are shown in Fig. 3f. The percentage of healthy vessels gradually declined down to only $4 \% 24 \mathrm{~h}$ p.i. Conversely, congestion and vascular rupture progressively increased with time. Interestingly, a significant increase in vascular congestion was observed as early as $3 \mathrm{~h}$ p.i. and thus before recruitment of neutrophils. The percentage of congested vessels continued to increase to reach a maximum percentage of $70 \%$ at $16 \mathrm{~h}$ p.i., which remained stable until $24 \mathrm{~h}$ p.i. A similar situation occurred with vascular rupture, with a slight increase at $6 \mathrm{~h}$ p.i. from 2 to $7 \%$ that progressively increased, reaching $25 \%$ at $16 \mathrm{~h}$ p.i.

Neutrophil depletion led to a decrease in the number of congested vessels and a concurrent increase in the occurrence of breached vessels with released red blood cells (Fig. $3 g$ and Supplementary Fig. 3e). To confirm and extend these results, vessel permeability to serum content was then measured using Evans Blue ${ }^{18}$. This dye was injected intravenously and $10 \mathrm{~min}$ post-injection, the circulating dye was removed from blood vessels by myocardial perfusion of heparin-containing buffer. As expected, a $24 \mathrm{~h}$ infection led to an increase of vascular permeability evidenced by high Evans blue accumulation in the tissue. In the absence of neutrophils, vascular leakage due to the infection was further increased (Fig. 3h). Altogether these data are in favor of a protective role for neutrophils during meningococcal infection. After being recruited, neutrophils incompletely control the number of bacteria in the infected vessels and thus partially reduce the vascular damage induced by the bacteria upon vascular colonization.

\footnotetext{
Ability of bacteria to colonize distinct vascular beds determine the level of neutrophil recruitment. Our results at this stage reveal a paradox, neutrophils are recruited upon vascular colonization and provide protection but fail to prevent the initiation and progression of the infection. A possible explanation of this incomplete neutrophil response is that the recruitment is insufficient in terms of number. This hypothesis is supported by the relatively low overall numbers of neutrophils $(93 \pm 27$ neutrophils $\mathrm{mm}^{-2}$ at $6 \mathrm{~h}$ p.i.) that are observed following $N$. meningitidis infection. As a comparison, TNFa stimulation or similar inflammatory situations can lead to about fivefold this value $^{19}$. We thus hypothesized that the unusual intraluminal
}

location of the bacteria, rather than the bacterium itself, is responsible for this moderate recruitment. To test this hypothesis, bacteria were injected directly into the xenograft intradermally and the inflammatory infiltrate was evaluated. The amounts of injected bacteria were adjusted to reach the amount found following intravascular infection (Fig. 4a). In these conditions, histological analysis of the human skin graft $3 \mathrm{~h}$ post intradermal infection showed a considerable infiltration of inflammatory cells in contrast to intravascular infection at the same time point (Fig. 4b). This was confirmed by flow cytometry analysis that highlighted a tenfold increase in neutrophil numbers as early as $3 \mathrm{~h}$ post intradermal infection (Fig. $4 \mathrm{c}$ ). Even at $6 \mathrm{~h}$ post intravascular infection, such numbers were not reached (Fig. 1c). These results point to the unexpectedly slow kinetics of neutrophil recruitment during vascular colonization and a particular impact of the vessel intraluminal location of the bacteria during infection.

It is important to note that the numbers given above represent average values throughout the tissue that could be highly heterogeneous and mask specific locations. We thus analyzed in-depth whether bacteria equally infected distinct types of vessels. For this, infected vessels were categorized into three classes: capillaries, venules, and arterioles. The distinction between human venules and arterioles was based on a combination of parameters: morphology, the intensity of the UEA-1 lectin staining ${ }^{20}$, intensity and direction of blood flow, as well as an arteriole-specific staining approach using Hydrazide AlexaFluor633, as previously described ${ }^{21}$ (Supplementary Fig. 4a, b). Capillaries were characterized by a luminal diameter below or equal to $10 \mu \mathrm{m}$. Remarkably, this classification revealed that $N$. meningitidis could infect all vessel types (Fig. 4d). Observed infected venules and arterioles had a similar diameter, $43.73 \pm$ $7.52 \mu \mathrm{m}$ and $49.13 \pm 3.77 \mu \mathrm{m}$, respectively (Fig. $4 \mathrm{e}$ ). In order to validate these observations made in an experimental mouse model, evidence of colonization of venules and arterioles in a human case were sought. Postmortem samples from a case of purpura fulminans were immuno-stained to visualize bacteria and tissue organization. Evidence of colonization of both venules and arterioles could be found in different tissues, such as in the liver and choroid plexus, in which aggregates of bacteria were associated with the endothelium (Fig. 4f, red arrows). $N$. meningitidis is thus able to colonize all types of vascular beds both in an experimental system and during human infections.

Neutrophil rolling was shown to preferentially occur on the venular endothelium but to a much lesser extent on arterioles and capillaries $^{22}$. We, therefore, hypothesized that they might not be 
a

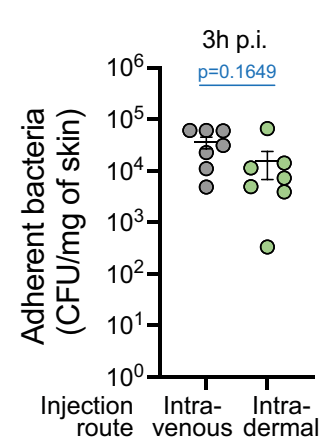

b

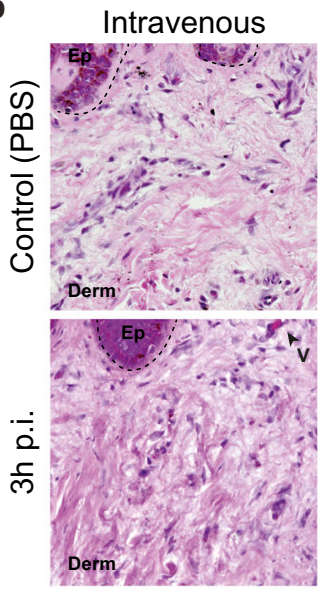

d
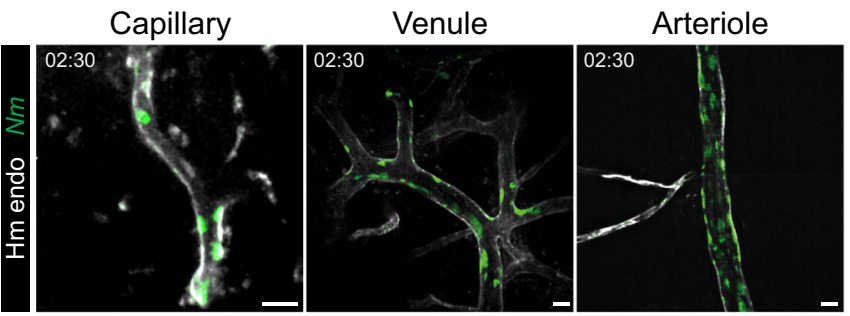

Arterioles

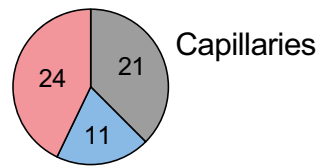

C
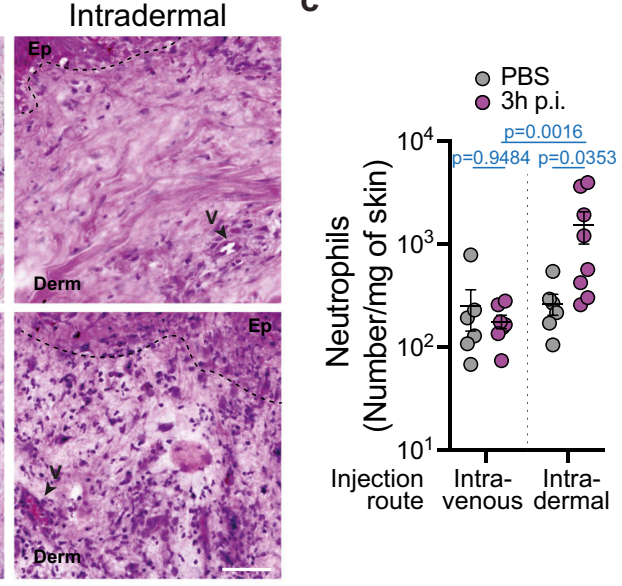

e

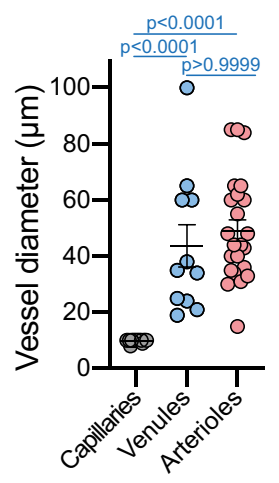

f

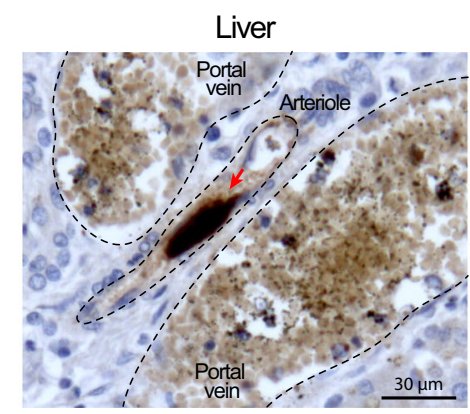

g

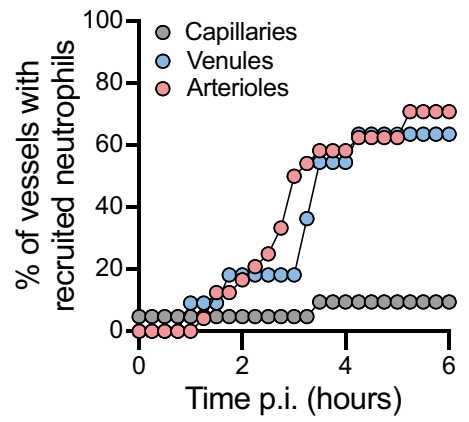

efficiently recruited to bacteria colonies localized in arterioles and capillaries. To test this hypothesis, the recruitment of neutrophils to the different vessel beds was evaluated. Neutrophils were rarely recruited to infected capillaries (Fig. 4g). The kinetics and percentage of infected vessels recruiting at least a single neutrophil were similar between venules and arterioles showing that some level of recruitment occurred in both of these vessel

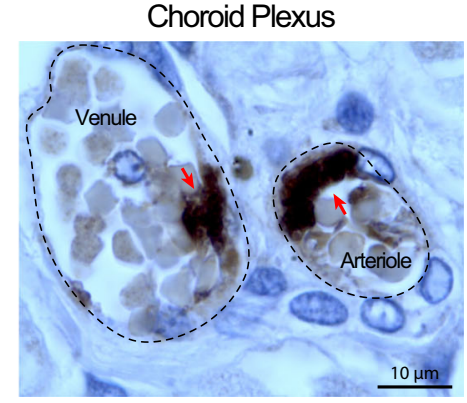

h

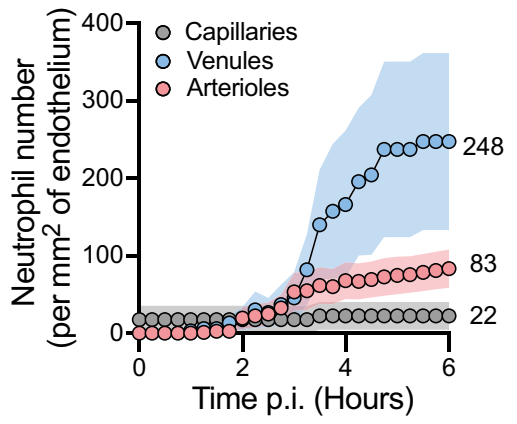

types (Fig. 4g). Importantly, however, the number of neutrophils recruited was much higher in venules than in arterioles, with 248 \pm 115 versus $83 \pm 25$ neutrophils per $\mathrm{mm}^{2}$ of the endothelium, respectively, at $6 \mathrm{~h}$ p.i. (Fig. $4 \mathrm{~h}$ ). The number of neutrophils recruited to capillaries was even lower than in arterioles $(22 \pm 18$ neutrophils per $\mathrm{mm}^{2}$ of endothelium). The ability of meningococci to colonize arterioles could thus provide an environment 
Fig. 4 Different types of vessels are infected with different levels of neutrophil recruitment. a Bacterial CFU counts from dissociated xenografts collected from intravenously (gray circles) or intradermally (green circles) infected mice for $3 \mathrm{~h}$. Two-tailed Mann-Whitney test. $n=7$ mice per condition, pooled from $N=3$ independent experiments. $\mathbf{b}$ Representative images of Haematoxylin and Eosin stain of xenografts harvested from mice $3 \mathrm{~h}$ following intravenous or intradermal injection of PBS (control) or Neisseria meningitidis ( $3 \mathrm{~h} \mathrm{p.i.).} \mathrm{Pictures} \mathrm{shown} \mathrm{are} \mathrm{representative} \mathrm{of} N=3$ mice per condition. Scale bar, $50 \mu \mathrm{m}$. The dashed lines delineate the epidermis/dermis border. Arrowheads indicate blood vessels. $V$ vessel. c Neutrophil numbers in xenografts of control (gray circles) and infected ( $3 \mathrm{~h} \mathrm{p.i.,} \mathrm{purple} \mathrm{circles)} \mathrm{mice} \mathrm{by} \mathrm{intravenous} \mathrm{or} \mathrm{intradermal} \mathrm{injection.} \mathrm{Two-tailed} \mathrm{Kruskal-Wallis} \mathrm{test} \mathrm{with} \mathrm{Dunn's}$ correction. $n=6$ mice (i.v. PBS and i.d. PBS), 7 mice (i.v. Infected), and 8 mice (i.d. infected), pooled from $N=3$ independent experiments.

d Representative images (maximum intensity z-projection) of vascular colonization $2 \mathrm{~h} 30$ post infection of the different vascular beds: capillaries, venules, and arterioles. The pie chart highlights the proportion and number of each vessel type. Scale bar, $20 \mu \mathrm{m}$. e Quantification of capillary, venule, and arteriole diameter. Two-tailed Kruskal-Wallis test with Dunn's correction. $\mathbf{f}$ Immunohistochemistry analysis of a human case of meningococcal septic shock showing vascular colonization by N. meningitidis in both venules and arterioles in the liver (left) and the choroid plexus (right). Bacteria were labeled using a polyclonal antibody directed against the strain cultured from the blood of this patient, as previously described ${ }^{28}$. Pictures shown are representative of one single human donor. $\mathbf{g}$ Percentage of vessels recruiting at least one neutrophil following infection according to the different vascular beds: capillaries (gray circles), venules (light-blue circles), and arterioles (light-red circles). $\mathbf{h}$ Numbers of neutrophils per square millimeter of endothelium according to the different vascular beds during the first $6 \mathrm{~h}$ of the infection. $\mathbf{a}, \mathbf{c}, \mathbf{e}, \mathbf{h}$ Data are shown as the mean \pm SEM. $\mathbf{d}, \mathbf{e}, \mathbf{g}, \mathbf{h}$ Quantifications were performed on $n=56$ vessels (21 capillaries, 11 venules, and 24 arterioles), pooled from $N=7$ infected mice imaged independently.

with lower numbers of neutrophils and thus an edge over the innate immune system.

\section{Neutrophils recruited to venules efficiently phagocytose bacteria.} The results described above show preferential recruitment of neutrophils to infected venules. We thus wondered whether neutrophils could have efficient bacterial killing properties in this context. The recruitment of neutrophils to infected venules was therefore explored in further detail in terms of location, kinetics, and phagocytic activity. Dynamic intravital imaging of infected venules revealed the expected progressive accumulation of bacteria along the vascular walls (Fig. 5a). Quantitative analysis of 11 venules revealed that after $4 \mathrm{~h}$ of infection, $55 \%$ of infected venules displayed neutrophil recruitment inside the vascular lumen (Fig. 5b). Rarely, neutrophils could be found in a perivascular location (1/11 venules). The numbers of intraluminal neutrophils reached $216 \pm 91$ neutrophils per $\mathrm{mm}^{2}$ of the endothelium after $6 \mathrm{~h}$ of infection (Fig. 5c). Neutrophils on the endothelium were highly motile and efficiently explored the infected endothelium by crawling on its surface (Supplementary Movie 4). On frequent occasions, neutrophils crawled in the direction of adherent bacterial aggregates. Neutrophils could efficiently internalize the adherent bacteria and thus detach them from the cellular surface (Fig. $5 \mathrm{~d}$ and Supplementary Movie 4). During long-term observations, bacterial aggregates initially adhering to the vascular wall could no longer be found following the arrival of neutrophils, demonstrating their efficiency in this context (Fig. 5a, red arrows and Supplementary Movie 4). This implies that neutrophils have the ability to detach bacteria tightly bound to the endothelial surface. Collectively, these experimental and clinical observations suggest that meningococci can adhere to venules and that neutrophils are readily recruited to these sites where they can efficiently phagocytose bacteria adhering to the endothelial wall.

As seen with other inflammatory stimuli ${ }^{22}$, our results show that in the context of arterioles only few neutrophils were recruited following $N$. meningitidis infection, leading to the hypothesis that the neutrophil response would be inefficient in this vessel type. This is striking considering the massive infection observed in arterioles. The recruitment of neutrophils to arterioles was then examined in detail. Intravital imaging revealed that the few neutrophils recruited to arterioles were either located in an intra- or extraluminal location, occasionally both (Fig. 6a). Neutrophil location was quantified in 24 arterioles over time, revealing that intraluminal and extraluminal or perivascular locations were represented in similar proportions (Fig. 6b). During the course of the infection, the recruitment of both intraluminal and perivascular neutrophils progressively increased over time with similar kinetics and similarly low numbers per surface of the endothelium (Fig. 6c). The few intraluminal neutrophils were frequently found to contain large amounts of bacteria (Fig. 6a, red arrows). In contrast, perivascular neutrophils were never observed to contain bacteria and even though neutrophils were often closely wrapped around the vascular wall (Supplementary Movie 5). No evidence of perivascular neutrophils translocating into infected arterioles was observed. Following 4-6h of infection, vessels were clogged with bacteria and intraluminal neutrophils progressively showed reduced motility (Fig. 6d and Supplementary Movie 6). These results show that when arterioles are infected, only limited amounts of neutrophils are recruited, and furthermore, their efficiency is limited. The few intravascular neutrophils are rapidly overwhelmed with bacteria filling the vessel lumen. Extravascular neutrophils fail to enter vessels and access bacteria.

These results were confirmed with a different meningococcal strain (strain 7761) belonging to serogroup B (Supplementary Fig. 5). As observed with the 8013 serogroup C strain used so far in this study, colonization occurred in different vascular beds and neutrophils were also recruited in venules but not in capillaries or arteries (Supplementary Fig. 5c). Therefore, the ability of meningococci to adhere to different vascular beds and more specifically arterioles and capillaries allow them to rapidly colonize these vessels before an efficient innate immune response can be mounted.

Despite intense colonization, endothelia lining capillaries and arterioles fail to upregulate inflammatory adhesion molecules. The above results raised the question of why neutrophils were efficiently recruited to venules but not to capillaries and arterioles despite the large accumulation of bacteria in all these vessel types. During infections caused by other pathogens, occurring inside tissues, neutrophils specifically exit the circulation through venules to reach the infection site. In this case, the specificity for venules is linked to the expression of a set of adhesion receptors on the endothelium surface, which are triggered by inflammatory signals coming from the infection site 22 . Although the intravascular location of the $N$. meningitidis infection generates a different situation, the difference in neutrophil response could be due to a differential expression of adhesion receptors. We first determined the consequence of meningococcal infection on the expression of a selection of neutrophil adhesion receptors on human umbilical vein endothelial cells in vitro. While ICAM-1 and VCAM- 1 cell surface levels barely increase $5 \mathrm{~h}$ after infection, the expression of E-selectin (CD62E) was strongly induced by the infection (Fig. 7a), as previously reported ${ }^{23}$. Expression of E- 

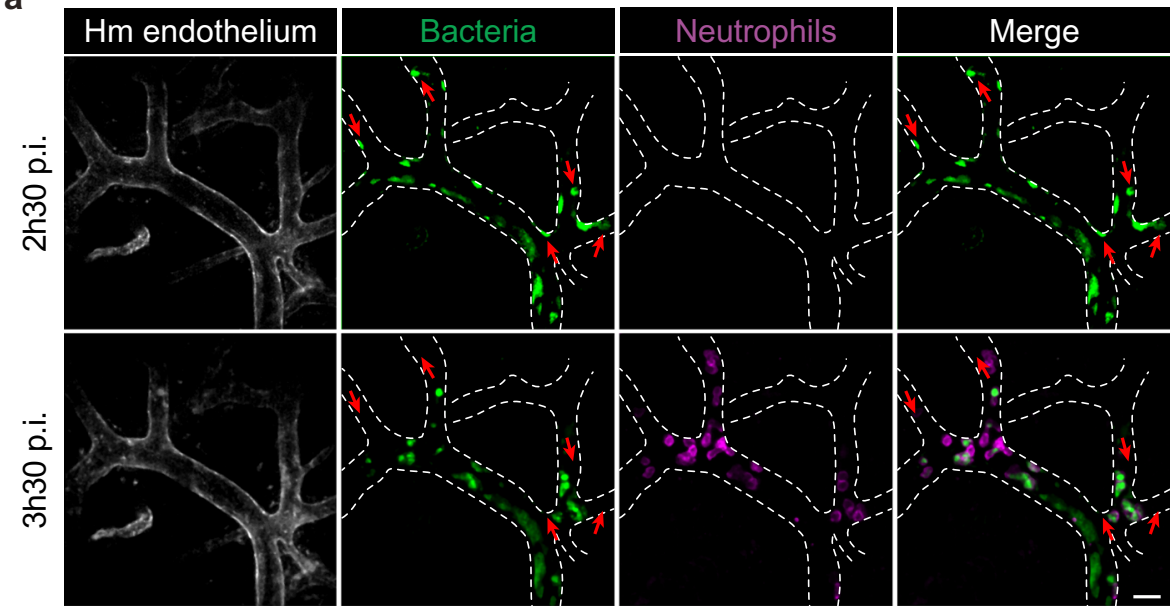

b

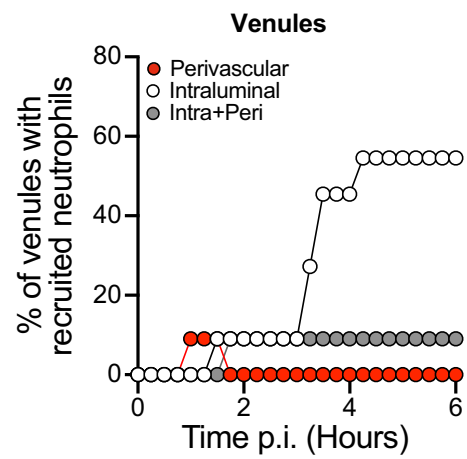

C

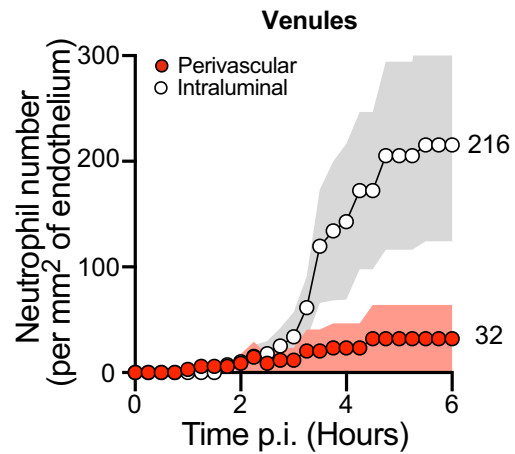

d

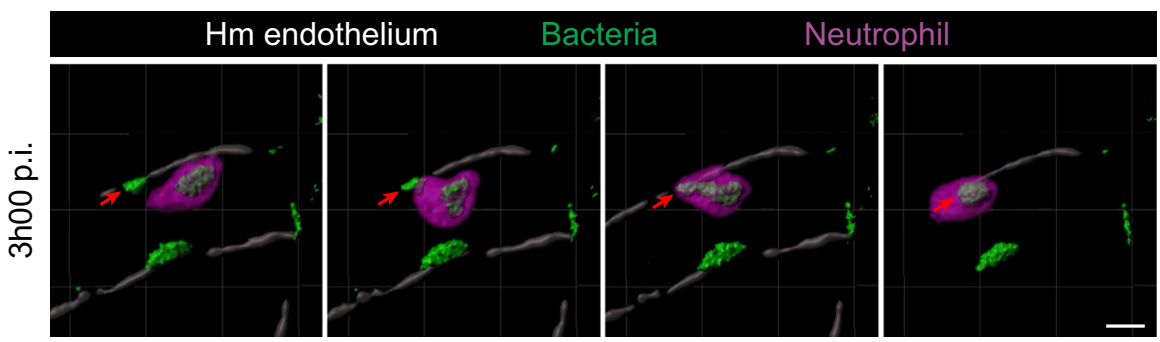

Fig. 5 Vascular colonization of venules leads to efficient neutrophil recruitment and function. a Intravital imaging (maximum intensity z-projection) of neutrophil (Ly-6G, magenta) recruitment to infected venules with iRFP-expressing Neisseria meningitidis (green). The human vessels are shown in gray (UEA-1 lectin) and dashed lines. Scale bars, $30 \mu \mathrm{m}$. b Percentage of venules recruiting only intraluminal (white circles), only perivascular (red circles), or both intraluminal and perivascular (gray circles) neutrophils. c Numbers of intraluminal and perivascular neutrophils per square millimeter of venular endothelium during the first $6 \mathrm{~h}$ of the infection. Data are shown as the mean \pm SEM. b, c Quantifications were performed on $n=11$ vessels, in total, pooled from $N=7$ infected mice imaged independently. d 3D-rendering of intravital imaging showing neutrophil (Ly-6G, magenta) migration toward and engulfing an adherent bacterial aggregate within a venule $3 \mathrm{~h}$ post infection with iRFP-expressing Neisseria meningitidis (green). The human venule is shown in gray (UEA-1 lectin). Scale bar, $10 \mu \mathrm{m}$.

selectin was highest at $5 \mathrm{~h}$ post infection, but was clearly detectable as early as $2 \mathrm{~h}$ post infection. This kinetics of expression being compatible with the kinetics of neutrophils recruitment observed during infection in vivo, we then determined the expression of E-selectin in the humanized mouse model of $N$. meningitidis infection. Low concentrations of fluorescently labeled anti-human CD62E monoclonal antibodies were introduced in the circulation of infected animals and accumulation of signals along infected vessel walls followed. Infection in the context of capillaries and arterioles did not lead to any specific signal (Fig. 7b), where only a low level and constant background signal could be seen. In contrast, infected venules showed a gradual accumulation of E-selectin signal during the course of the infection (Fig. 7b). These results are consistent with previous studies using TNFa or LPS stimulation ${ }^{24-26}$. Interestingly, the signal was heterogeneous along the human endothelium surface and strongly colocalized with bacterial colonies (Fig. 7c). Signals leading to E-selectin expression thus had a local component linked to bacterial adhesion. The role of E-selectin in N. meningitidis-induced neutrophil recruitment was then explored in vivo using a blocking antibody. As expected ${ }^{27}$, neutrophil recruitment was strongly impaired in venules upon E-selectin blocking $(47 \pm 27$ neutrophils versus $188 \pm 53$ neutrophils in the isotype control condition), while robust vascular colonization was taking place (Fig. 7d, e). E-selectin is thus a central player in the recruitment of neutrophils to venules following $N$. meningitidis infection.

Together, these results show that despite massive infection and in contrast to the venular endothelium, endothelia from arterioles and capillaries fail to express E-selectin on their surface upon infection, thus preferentially targeting neutrophil 
a

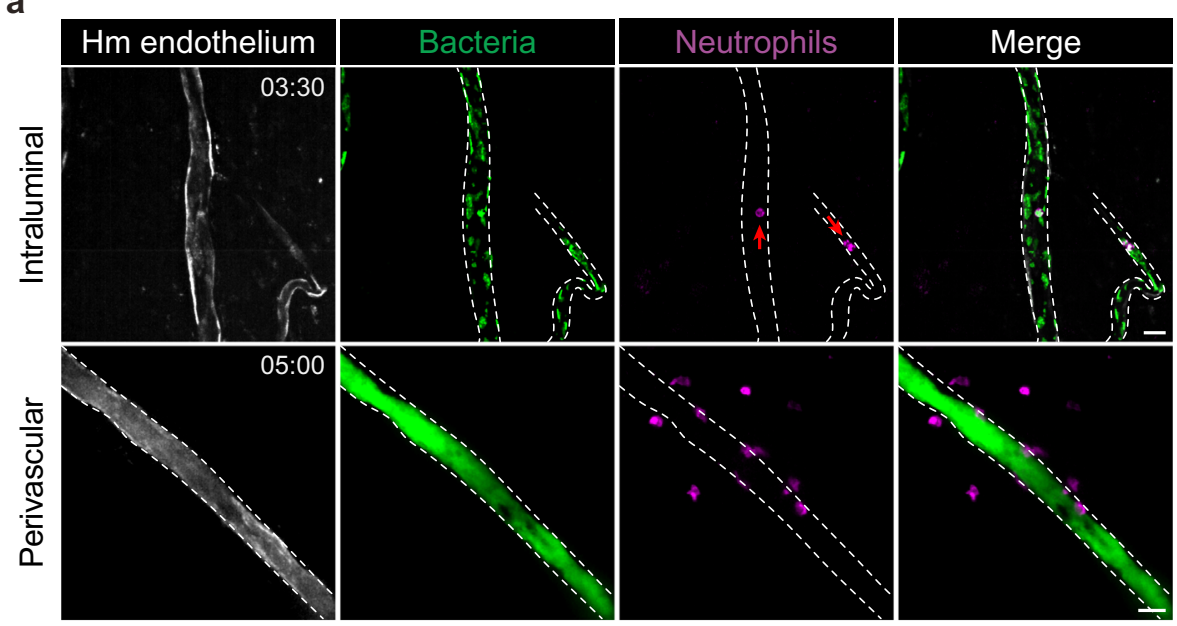

b

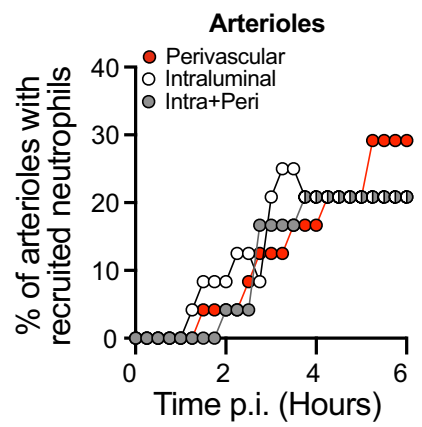

C

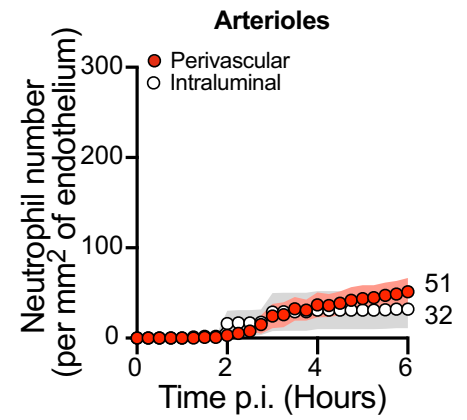

d

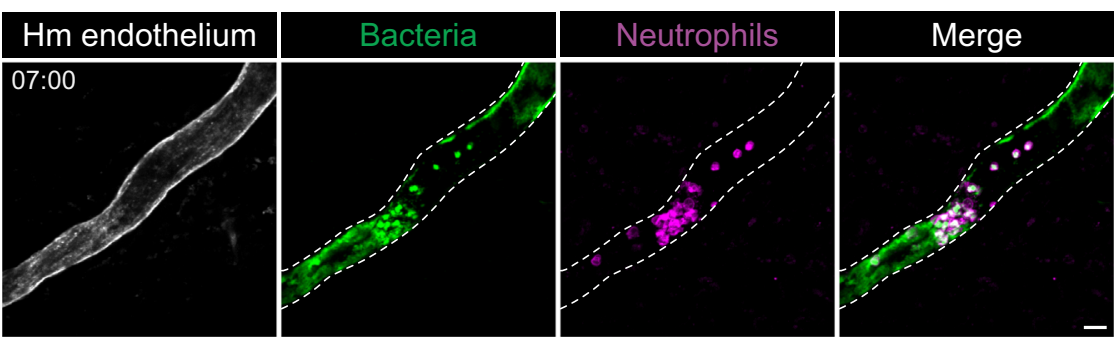

Fig. 6 Vascular colonization of arterioles leads to limited neutrophil recruitment and efficiency. a Intravital imaging (maximum intensity z-projection) of intraluminal (top panel) or perivascular (bottom panel) recruitment of neutrophils (Ly-6G, magenta) to arterioles 3-5 h post infection with iRFP-expressing Neisseria meningitidis (green). The human vessels are shown in gray (UEA-1 lectin) and dashed lines. Time, hh:min. Scale bar, $30 \mu \mathrm{m}$. b Percentage of arterioles recruiting only intraluminal (white circles), only perivascular (red circles), or both intraluminal and perivascular (gray circles) neutrophils. c Numbers of intraluminal and perivascular neutrophils per square millimeter of arteriolar endothelium during the first $6 \mathrm{~h}$ of the infection. Data are shown as the mean \pm SEM. b, c Quantifications were performed on $n=24$ vessels, in total, pooled from $N=7$ infected mice imaged independently. $\mathbf{d}$ Intravital imaging (maximum intensity z-projection) of neutrophil (Ly-6G, magenta) entrapped within bacterial aggregates within an infected arteriole $7 \mathrm{~h}$ post infection with iRFP-expressing Neisseria meningitidis (green). The human vessels are shown in gray (UEA-1 lectin) and dashed lines. Scale bar, $30 \mu \mathrm{m}$.

recruitment towards infected venules over arterioles and capillaries.

\section{Discussion}

To mount a protective innate immune response, the inflammatory cascade needs to trigger the recruitment of numerous neutrophils to the precise site of infection, leading to the efficient clearance of the pathogen. In the case of fulminant infections caused by meningococci, the innate immune response is not successful at keeping the pathogen in check. Experimental results provided here, confirmed by observations in human cases, explain how this is due to the particular proliferation niche of this bacterium. While meningococci have the ability to colonize different vascular beds, capillaries, venules, and arterioles, only infected venules allow efficient neutrophil recruitment.
The ability of bacteria to adhere to the endothelium wall is the starting point for the innate immune response as this allows the concentration of bacteria at a specific focal point rather than their systemic distribution in the blood. In absence of adhesion, neutrophil recruitment is barely detectable. Bacterial accumulation on the endothelial surface is thus a trigger for neutrophil recruitment. The ability of bacteria to autoaggregate also enhances neutrophil recruitment. This is likely explained by the amplification of bacterial numbers at the site of infection due to the three-dimensional accumulation of bacteria. It could also be envisioned that the formation of aggregates leads to more perturbations in blood flow and subsequently more inflammatory signals. Nevertheless, type IV pili are central players in triggering the inflammatory response by allowing adhesion and autoaggregation. Bacterial adhesion also shapes the innate immune 
a

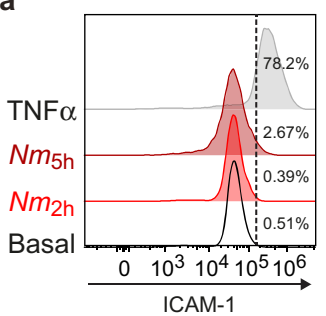

b
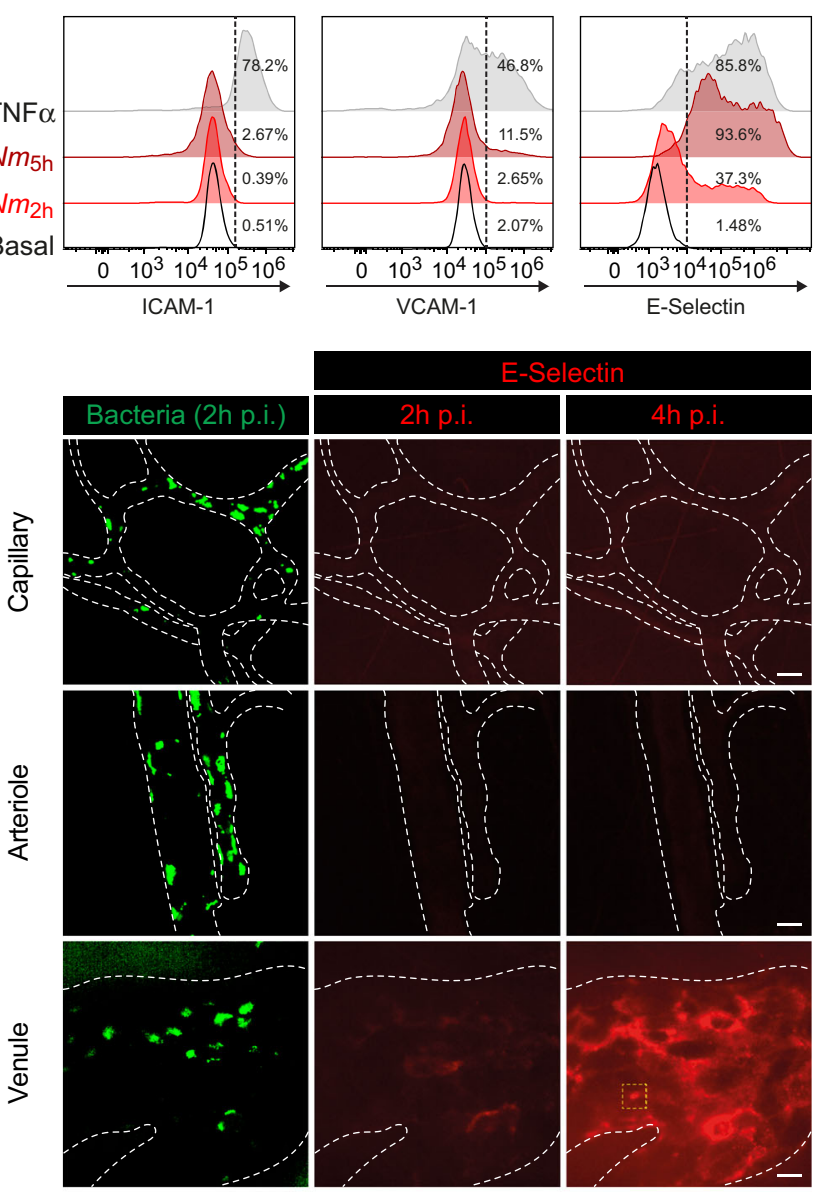

C

$\frac{0}{\frac{0}{0}}$
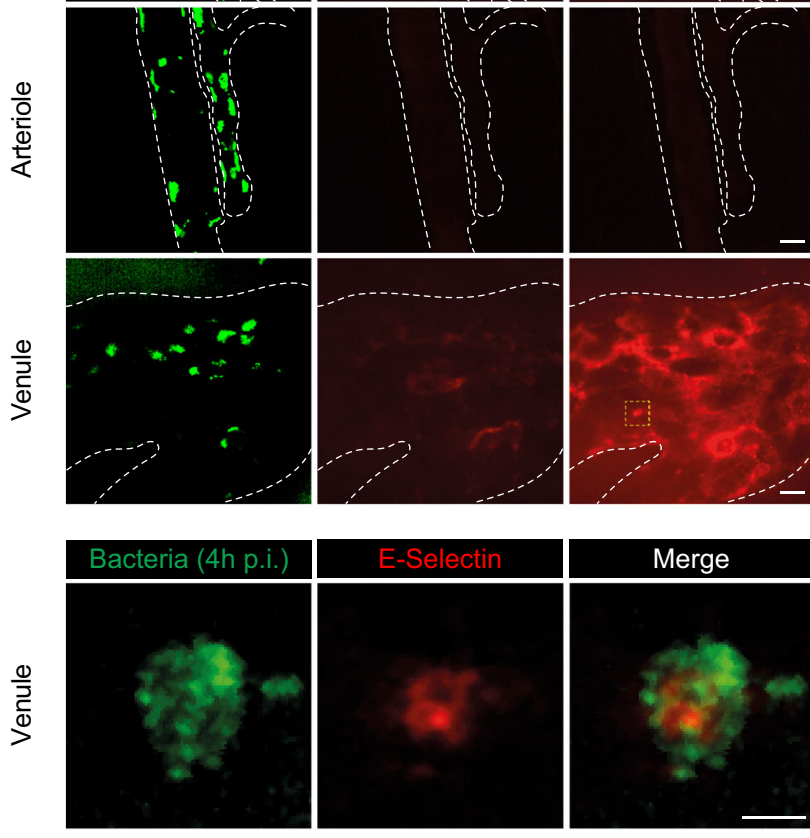

d

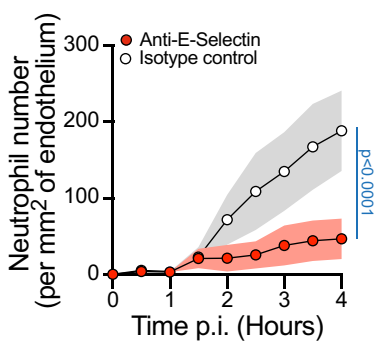

e

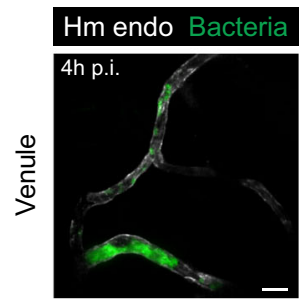

response by leading to the colonization of different vessel types including capillaries. In a previous study focusing on meningitis and infection of the brain, we had shown preferential colonization of capillaries in this organ ${ }^{28}$. This study, based on an in vivo model and clinical samples, extends these findings to other organs, confirms infection of capillaries, and shows that other vascular beds can be infected.

Venules are readily infected and bacterial aggregates bound to the venular endothelial surface can be seen $2-3 \mathrm{~h}$ p.i. In this context, neutrophils are recruited rapidly and in large numbers at similar times. Neutrophil recruitment correlates with the expression of E-selectin at the surface of the venular endothelium,
Fig. 7 E-selectin endothelium surface expression is differentially upregulated according to the vascular bed upon infection. a Flow cytometry analysis of cell surface expression of ICAM-1, VCAM-1, and Eselectin (CD62E) on HUVEC cells under resting conditions (Basal, open histograms) or following infection with Neisseria meningitidis for $2 \mathrm{~h}\left(\mathrm{Nm}_{2 \mathrm{~h}}\right.$, red histograms) or $5 \mathrm{~h}$ ( $\mathrm{Nm}_{5 \mathrm{~h}}$, dark-red histograms) or an overnight incubation with $20 \mathrm{ng} \mathrm{ml}^{-1} \mathrm{TNF} \alpha$ gray histograms). Data are representatives of $N=3$ independent experiments. The percentages of positive cells (above the dashed lines) are shown per condition and marker. $\mathbf{b}$ In vivo expression of E-selectin at the surface of different human vessel types (capillary, arteriole, and venule) following two ( $2 \mathrm{~h} \mathrm{p.i.)}$ and four ( $4 \mathrm{~h} \mathrm{p.i.)} \mathrm{hours} \mathrm{of}$ infection. Images (maximum intensity z-projection) are representative of $N$ $=3$ infected mice imaged independently. GFP-expressing Neisseria meningitidis appears in green and E-selectin in red following in vivo labeling by i.v. injection of PE-labeled anti-CD62E monoclonal antibody. Dashed lines delineate human vessels (UEA-1 lectin). Scale bar, $20 \mu \mathrm{m}$. c Highmagnification view (yellow dashed square in panel $\mathbf{b}$ ) of a bacterial microcolony at $4 \mathrm{~h}$ p.i. on the venular endothelium surface and the local upregulation of E-selectin expression. Scale bar, $5 \mu \mathrm{m}$.

d Movies obtained from intravital imaging were used to quantify the numbers of neutrophils per square millimeter of venular endothelium during the first $4 \mathrm{~h}$ of the infection in presence of anti-E-selectin blocking antibody (red circles) or isotype control (white circles). Data are shown as the mean \pm SEM. Quantifications were performed on $n=22$ and 8 venules for $E$ selectin blocking and isotype control conditions, respectively, pooled from $N$ $=3$ infected mice imaged independently per condition. Two-tailed two-way ANOVA. e Representative image (maximum intensity z-projection) of venular colonization $4 \mathrm{~h}$ post infection by Neisseria meningitidis (green) in presence of the anti-E-selectin blocking antibody. The human endothelium was labeled using UEA-1 lectin (gray). The picture shown is representative of $N=3$ mice imaged independently. Scale bar, $50 \mu \mathrm{m}$.

which promotes neutrophil adhesion and crawling. Upon venular infection, neutrophils are located inside the vascular lumen, actively crawl on the endothelium surface, and phagocytose bacteria. Evidence of the disappearance of aggregates from the endothelium surface upon neutrophil local recruitment is clearly seen, thus demonstrating their functional impact on bacterial clearance. Accordingly, depletion of neutrophils leads to more severe infection as measured by global bacterial numbers inside the tissue and evidence of vascular damage. Interestingly, at early time points post infection, high-resolution intravital microscopy of infected venules allowed direct observation of adhering bacteria being engulfed by neutrophils. Considering the intimate type IV pili-mediated adhesion of bacteria to the endothelial surface, allowing bacterial aggregates to resist high mechanical stress ${ }^{29}$, the phagocytosis of such bacterial colonies by neutrophils thus implies the engagement of intense forces that would warrant further investigation.

The scenario in arterioles and capillaries is very different as bacterial elimination is made difficult by several factors. Bacterial proliferation is barely altered by very limited numbers of recruited neutrophils, if any. The absence of endothelial adhesion receptor expression, such as E-selectin, in these vascular beds upon infection, provides an explanation for the reduced levels of intravascular neutrophil recruitment. At later time points post infection, vessels are entirely full of bacteria, to the point where the few neutrophils on site are trapped in bacterial colonies. The combination of coagulation and occupation of the luminal space by bacteria and congested red blood cells rapidly stops blood flow and consequently, neutrophils have limited access to reach the bacterial colonies. The rare neutrophils recruited following capillary infection were intravascular. In the case of arterioles, neutrophils can also be found surrounding vessels in the animal 
model as observed in the human cases, confirming the relevance of these observations ${ }^{7}$. Such neutrophils likely extravasated from nearby venules and migrated through the tissue toward the infected arterioles. Interestingly, these extraluminal neutrophils fail to enter arterioles from the outside and remain within the perivascular area for hours without any evidence of phagocytosis. Although a matter of debate, the ability of neutrophils within tissues to go back inside venules by a process termed reverse migration has been described ${ }^{30}$. In the case of meningococcal infections, the inability of these cells to enter vessels or even reach inside infected arterioles could simply be explained by the nature and thickness of the arteriolar vascular wall. It should be pointed out that the recruitment of neutrophils around arterioles can be seen at $6 \mathrm{~h}$ p.i. when there is now evidence of vascular leakage. The lumen of arterioles and capillaries thus provide a safe haven for the bacterium.

The question remains as to why intraluminal infection of arterioles is insufficient to induce the expression of adhesion receptors despite intense local infection. In arterioles, it is generally accepted that the expression of such adhesion molecules is lower than in venules, leading to lower levels of interaction of neutrophils with the arteriolar endothelium ${ }^{31}$. Nevertheless, in certain instances, the expression of adhesion molecules in arterioles is significantly increased during inflammation. For instance, $\mathrm{TNF} \alpha$ treatment results in increased expression of P- and Eselectin, as well as ICAM-1 and VCAM-1, in cremaster muscle arterioles $^{32}$. Angiotensin II has also been reported to induce the recruitment of neutrophils to arterioles ${ }^{33}$. It nevertheless remains that during $N$. meningitidis infection, despite the impressive accumulation of bacteria within the lumen of arterioles, insufficient levels of adhesion molecules are expressed to provoke the efficient recruitment of neutrophils.

The ability of bacteria to escape the innate immune response and accumulate inside vessels is crucial in triggering the vascular damages that constitute the hallmark of this disease. They include vascular congestions, intravascular coagulation, and loss of vessel integrity. The rapid and massive occurrence of these damages lead to decreased tissue oxygenation and finally organ dysfunction. In addition to determining the role of neutrophils in this process, this study provides a detailed description of the kinetics of bacteria-induced vascular damages. At $2-3 \mathrm{~h}$ post infection, $\sim 30 \%$ of vessels in the infected tissue show evidence of congestion on histological slides. At these early time points, barely any neutrophils are recruited, suggesting that they have little or no role in the initial stages of vascular damage. At later stages (16-24 h), nearly all vessels of the infected tissue become congested providing a striking illustration of how infection causes organ failure. At these same time points, a fraction of vessels lose their integrity and vascular contents are released in the tissue. Our study shows that neutrophils play a protective role in this second phase of the infection by limiting the extent of vascular leakage. This is associated with increased congestion, suggesting that neutrophils could enhance coagulation. Exactly how neutrophils limit vascular damage remains to be determined. Assuming that the bacteria themselves trigger the vascular damage, the effect of neutrophils on the number of bacteria could be sufficient to explain their protective role on the vessels. Alternatively, neutrophils could facilitate the coagulation response as previously seen in other models ${ }^{34}$. The specific impact of neutrophils on coagulation during $N$. meningitidis infection needs to be further explored as it could potentially limit further colonization by bacteria.

The results of this study provide an explanation of the fulminant progression of $N$. meningitidis infection by showing how the adhesive and aggregative properties of the bacteria protect them from the function of the innate immune cells. Meningococci occupy the vascular lumen of different vascular beds and most importantly arterioles and capillaries to which neutrophils are poorly recruited following infection. As a consequence, the uncontrolled accumulation of bacteria inside such vessels leads to vascular damages that characterize this fulminant life-threatening infection. This particular means of evading the immune response by targeting a form of immune-privileged site could be involved in the high mortality still associated with purpura fulminans ${ }^{35}$.

\section{Methods}

Mice. SCID/Beige (CB17.Cg-Prkdcscid Lyst $\left.t^{b-J} / \mathrm{Crl}\right)$ mice, which were used in all the experiments performed in this study except for the intravital imaging of neutrophils during the late phase of the infection, were purchased from Charles Rivers (France). $\operatorname{Rag}_{2}{ }^{-/-} \gamma_{c}{ }^{-l}$ mice (kindly provided by the Central Animal Facility, Institut Pasteur, Paris, France) were bred with $L y s M g f p /+$ mice (kindly provided by Ivo Gomperts Boneca, Institut Pasteur, Paris, France) to obtain $\mathrm{Rag}_{2}{ }^{-/-} \gamma_{c}{ }^{-/-} L y s M^{g} \mathrm{~g} p /+$ mice that were used for the intravital imaging of neutrophils during the late phase of the infection. All mouse strains were housed under the specific pathogen-free condition at Institut Pasteur. Mice were kept under standard conditions (light 07.00-19.00 h; temperature $22 \pm 1{ }^{\circ} \mathrm{C}$; humidity $50 \pm 10 \%$ ) and received sterilized rodent feed and water ad libitum. All experiments were performed in agreement with guidelines established by the French and European regulations for the care and use of laboratory animals and approved by the Institut Pasteur Committee on Animal Welfare (CETEA) under the protocol code CETEA 2015-0025. For all experiments, male and female mice between 6 and 10 weeks of age were used. Littermates were randomly assigned to experimental groups.

Human skin. Normal human skin was obtained from adult patients (20-60 years old), both males and females, undergoing plastic surgery in the service de Chirurgie Reconstructrice et Plastique of Groupe Hospitalier Saint Joseph (Paris, France) or the service de Chirurgie Plastique, Recontructrice et Esthetique of Hôpital Européen Georges Pompidou (Paris, France). In accordance with the French legislation, patients were informed and did not refuse to participate in the study. All procedures were approved by the local ethical committee Comité d'Evaluation Ethique de l'INSERM IRB 00003888 FWA 00005881, Paris, France Opinion: 11048 .

Xenograft model of infection. Five to eight weeks old mice, both males and females, were grafted with human skin as previously described ${ }^{8}$. Briefly, a graft bed of $\sim 1-2 \mathrm{~cm}^{2}$ was prepared on the flank of anesthetized mice (intraperitoneal injection of ketamine and xylazine at $100 \mathrm{mg} \mathrm{kg}^{-1}$ and $8.5 \mathrm{mg} \mathrm{kg}^{-1}$, respectively) by removing the mouse epithelium and the upper dermis layer. A human skin graft (200- $\mu \mathrm{m}$ thick) comprising the human epidermis and the papillary dermis was immediately placed over the graft bed. Grafts were fixed in place with surgical glue (Vetbond, 3 M, USA) and dressings were applied for 2 weeks. Grafted mice were used for experimentation 3-6 weeks post surgery when the human dermal microvasculature is anastomosed to the mouse circulation without evidence of local inflammation, as previously described ${ }^{8}$. All efforts were made to minimize suffering.

Neisseria meningitidis strains and mouse infection. Most of the experiments described in this study were performed using $N$. meningitidis strains derived from the recently sequenced 8013 serogroup C strain (http://www.genoscope.cns.fr/agc/ nemesys) ${ }^{36}$, except when mentioned. Mutations in pilD and pilC1 genes have been previously described ${ }^{36,37}$. Wild-type (SB), SA, pilD, and pilC1 bacterial strains genetically modified to constitutively express either the green fluorescent protein (GFP) or the near-infrared fluorescent protein (iRFP) under the control of the pilE gene promoter, were previously generated and described ${ }^{8,12,38}$. Strain 7761 used in Supplementary Fig. 5 is a Neisseria meningitidis serogroup B strain isolated from the blood of a 23-year-old patient at the Limoges University Hospital (France) in 2008. Strains were streaked from $-80^{\circ} \mathrm{C}$ freezer stock onto GCB agar plates and grown overnight in a moist atmosphere containing $5 \% \mathrm{CO}_{2}$ at $37^{\circ} \mathrm{C}$. For all experiments, bacteria were transferred to liquid cultures in pre-warmed RPMI1640 medium (Gibco) supplemented with $10 \% \mathrm{FBS}$ at adjusted $\mathrm{OD}_{600 \mathrm{~nm}}=0.05$, and incubated with gentle agitation for $2 \mathrm{~h}$ at $37^{\circ} \mathrm{C}$ in the presence of $5 \% \mathrm{CO}_{2}$. Bacteria were washed twice in PBS and resuspended to $10^{8} \mathrm{CFU} \mathrm{ml}^{-1}$ in $1 \times \mathrm{PBS}$. Prior to infection, mice were injected intraperitoneally with $8 \mathrm{mg}$ of human transferrin (Sigma Aldrich) to promote bacterial growth in vivo, as previously described $^{8}$. Mice were infected by intravenous injection of $100 \mu \mathrm{l}$ of the bacterial inoculum $\left(10^{7} \mathrm{CFU}\right.$ total). For intradermal infection, $10^{6}$ bacteria were resuspended in $50 \mu \mathrm{l} 1 \times$ PBS containing $8 \mathrm{mg}$ of human transferrin. No influence of mice sex on bacterial colonization has been observed (data not shown).

Cell culture and infection. Primary human umbilical endothelial cells (HUVECs) were purchased from Lonza (pooled donors, \# C2519A) and cultured in EGM-2 complete medium (Lonza, \# CC-3162) without antibiotics. Cells were used between passages 2 and 6 . For infection, $2.5 \times 10^{5}$ HUVECs were plated in wells of six-well 
plates and stimulated overnight with $20 \mathrm{ng} \mathrm{ml}^{-1}$ human recombinant TNFa (PeproTech/Tebu Bio, \#300-01A-A) or infected at MOI $=200$ with Neisseria meningitidis for $30 \mathrm{~min}$, washed to removed nonadherent bacteria and incubated for an additional $2 \mathrm{~h}$ or $5 \mathrm{~h}$, prior to be harvested and stained for flow cytometry analysis (see below).

Ex vivo colony-forming units (CFU) enumeration. To assess bacteremia (blood circulating bacteria) in infected animals, $10 \mu \mathrm{l}$ of blood was sampled before infection, $5 \mathrm{~min}$ after infection, and at the time of sacrifice. Serial dilutions of blood were plated on GCB agar plates and incubated overnight at $37^{\circ} \mathrm{C}$ and in a moist atmosphere containing $5 \% \mathrm{CO}_{2}$. Bacterial counts were expressed in colony-forming units (CFU) per $\mathrm{ml}$ of blood.

To assess the extent of vascular colonization by meningococci (adherent bacteria) following mouse sacrifice at indicated times post infection, tissue biopsies were collected using a sterile dermatological biopsy puncher $\left(\sim 4 \mathrm{~mm}^{2}\right)$, weighted and placed in $500 \mu \mathrm{l} 1 \times \mathrm{PBS}$. Skin biopsies were dissociated and homogenized using MagNA lyser homogenizer (Roche, France) and serial dilutions of skin homogenates were plated on GCB plates and incubated overnight at $37^{\circ} \mathrm{C}$ and in a moist atmosphere containing $5 \% \mathrm{CO}_{2}$. Bacterial counts were expressed in colonyforming units (CFU) per mg of the skin.

Cell dissociation from skin samples. Cell suspensions were isolated from human skin xenograft or mouse skin as previously described ${ }^{39}$ with some modifications: skin biopsies were collected immediately after mouse sacrifice and weighed on a fine balance. Subsequently, the skin was cut into small pieces and digested with gentle agitation for $60-90 \mathrm{~min}$ at $37^{\circ} \mathrm{C}$ in $\mathrm{CO}_{2}$-independent medium containing $25 \mathrm{mM}$ HEPES, $0.4 \mathrm{mg} \mathrm{ml}^{-1}$ Liberase TL (Sigma Aldrich), $0.04 \mathrm{mg} \mathrm{ml}^{-1}$ DNase I (Sigma Aldrich), and $100 \mathrm{U} \mathrm{ml}^{-1}$ penicillin/streptomycin (Gibco). The resulting single-cell suspension was passed through a $70-\mu \mathrm{m}$ cell strainer (BD Bioscience) and treated with $1 \times$ RBC lysis solution (BioLegend). Cells were again filtered using a $40-\mu \mathrm{m}$ cell strainer (BD Bioscience) and counted. Cell viability was determined using trypan blue exclusion.

\section{Flow cytometry}

Dissociated skin. Single-cell suspensions were labeled in aliquots of $10^{6}$ cells per $100 \mu \mathrm{l}$ in $1 \times$ PBS supplemented with $2 \%$ FBS, according to standard protocols ${ }^{40}$. Fc receptors were blocked using the anti-mouse CD16/CD32 (FcBlock clone 2.4G2) monoclonal antibodies (BD Biosciences, \#553141, 1/400), and cells were stained for $30 \mathrm{~min}$ at $4{ }^{\circ} \mathrm{C}$ with a combination of the following anti-mouse immunophenotyping antibodies: from Biolegend: PacificBlue-conjugated anti-Ly-6C (clone HK1.4, \#128014, 1/400), PacificBlue- or PE/Cy7-conjugated anti-Ly-6G/Ly-6C (GR-1) (clone RB6-8C5, \#108430 or \#108416, 1/400 and 1/200, respectively), PacificBlue-conjugated anti-CD45 (clone 30-F11, \#103126, 1/200). From eBioscience: APC-conjugated anti-CD11b (clone M1/70, \#17-0112-82, 1/200), PEconjugated anti-Ly-6C (clone HK1.4, \#12-5932-82, 1/400). From BD Biosciences: BUV395-conjugated anti-CD45 (clone 30-F11, \#564279, 1/200). Exclusion of nonviable cells has been achieved using eFluor780 Fixable viability dye (eBioscience, \#65-0865) according to the manufacturer's instructions. After staining, cells were washed in $1 \times$ PBS and fixed for $20 \mathrm{~min}$ at $4{ }^{\circ} \mathrm{C}$ with $4 \%$ paraformaldehyde in $1 \times$ PBS and washed with $1 \times$ PBS supplemented with $2 \%$ FBS. Data were acquired using a BD LSR Fortessa ${ }^{\mathrm{TM}}$ flow cytometer controlled with the BD FACSDiva software (BD Bioscience). Data analysis was carried out using FlowJo software v10 (Tree Star, Ashland, OR, USA). Numbers of cells were expressed per mg of the skin according to the following formula: $N=\frac{n(p / 100)}{W}$; where $N$ is the number of cells per milligram of skin, $n$ the number of live cells obtained after skin dissociation (Trypan blue exclusion), $P$ the percentage of the given cell population among the "live cells" gate determined by flow cytometry and $W$ the weight in milligram of the corresponding biopsy before skin dissociation.

Cultured endothelial cells. Following infection for the indicated times or TNFo treatment, cells were harvested using $37^{\circ} \mathrm{C}$ preheated Versene (Gibco, \#15040066) to preserve cell surface epitopes. Fc receptors were blocked using Human TruStain FcX (BioLegend, \#422302, 1/20) diluted in cold FACS buffer (1× PBS supplemented with $0.5 \%$ BSA and 2 mM EDTA). Cells were stained for $30 \mathrm{~min}$ on ice using AlexaFluor700-conjugated anti-human CD54/ICAM-1 (clone 1H4, Life Technologies, \#MA528553, 1/75), PE-Cy7-conjugated anti-human CD106/VCAM1 (clone STA, eBioscience, \#25-1069-42, 1/75), and PE-conjugated anti-human CD62E/E-selectin (clone P2H3, eBioscience, \#12-0627-42, 1/20) diluted in cold FACS buffer. Exclusion of nonviable cells has been achieved using eFluor506 Fixable viability dye (eBioscience, \# 65-0866-14) according to the manufacturer's instructions. After staining, cells were washed in cold $1 \times$ PBS and fixed overnight at $4{ }^{\circ} \mathrm{C}$ with $1 \%$ paraformaldehyde in $1 \times$ PBS. Data were acquired using a CytoFLEX LX flow cytometer controlled with the CytExpert Software (Beckman Coulter) and analysis was carried out using FlowJo software v10 (Tree Star, Ashland, OR, USA).

Measurement of soluble factors. Mechanically homogenized skin samples were filtered using Corning Costar Spin-X centrifuge tubes (pore size $0.22 \mu \mathrm{m}$ ). The resulting filtrates were used to assess the levels of both mouse and human inflammatory cytokines using Cytometric Bead Array according to the manufacturer's instructions (BD Bioscience, \#552364 and \#551811, respectively) on a BD LSR Fortessa flow cytometer. Data analyses were performed using the FCAP array software (BD Biosciences). Levels of mouse GRO alpha (KC/CXCL1) and MIP-2 (CXCL2) were measured using ProcartaPlex Mix\&Match Mouse kit (eBioscience) according to the manufacturer's instructions and detected using the Bioplex200 apparatus (Bio-Rad).

\section{Preparation of skin tissue samples for fluorescence microscopy and histology}

Skin biopsies were collected from control mice or at different times post infection fixed in $4 \%$ paraformaldehyde (PFA) for $24 \mathrm{~h}$ (or $2 \%$ PFA up to 15 days) at $4{ }^{\circ} \mathrm{C}$, washed at least twice in $1 \times$ PBS, and dehydrated overnight in $20 \%(\mathrm{w} / \mathrm{v})$ sucrose at $4{ }^{\circ} \mathrm{C}$. Tissue samples were then embedded in OCT (Tissuetek) and frozen at $-80^{\circ} \mathrm{C}$. In all, 7-10- $\mu \mathrm{m}$ thick cryostat sections were immobilized on Superfrost plus microscopy slides (Thermo Scientific) and prepared either for hematoxylin and eosin (H\&E) stain or immunostaining using the Mouse on Mouse kit (Vector Laboratories) according to the manufacturer's instructions. Sections were stained using the following antibodies: rat anti-mouse Ly-6C/Ly-6G (RB6-8C5, BD Bioscience, \#550291, 1/200) followed by AlexaFluor647-conjugated goat anti-rat IgG (ThermoFisher Scientific, \#A21247, 1/200). Human vessels were stained using Rhodamine-conjugated Ulex Europaeus Agglutinin I (UEA-1) lectin (Vector Laboratories, \#DL-1067, 1/200). Skin sections were then mounted using Vectashield mounting reagent (Vector Laboratories). Fluorescent images were acquired on an inverted spinning-disk confocal microscope (Ti-eclipse, Nikon) equipped with an EMCCD camera (Evolve, Photometrics) and a $\times 40$ oil immersion objective using Metamorph Imaging Software (Molecular Devices). Scanning of H\&E-stained cryosections was performed using a slide scanner Axio scan Z1 (Zeiss) at $\times 20$ magnification and images were processed using ZenBlue software (Zeiss). Image processing was performed using Fiji software ${ }^{41}$, except when mentioned. Images shown in the figures were cropped from large fields, rotated, and their contrast and brightness manually adjusted.

\section{Spinning-disk confocal intravital imaging}

Mouse surgery and preparation of the skin flap. Intravital imaging of the human xenograft was adapted from ref. ${ }^{20}$. Briefly, 30-min prior to surgery, mice were injected subcutaneously with buprenorphine $\left(0.05 \mathrm{mg} \mathrm{kg}^{-1}\right)$ and anesthetized by spontaneous inhalation of isoflurane in $100 \%$ oxygen (induction: $4 \%$; maintenance $1.5 \%$ at $0.3 \mathrm{~L} \mathrm{~min}^{-1}$ ). A middle dorsal incision was made from the neck to the lower back and the skin supporting the human xenograft was flipped and secured onto an aluminum custom-made heated deck $\left(36^{\circ} \mathrm{C}\right)$. The human microvasculature within the graft was exposed by carefully removing the excess of connective tissue. The skin flap was covered with a coverslip maintained thanks to a $3 \mathrm{D}$-printed custom-made holder to avoid any pressure on the xenograft vasculature, sealed with vacuum grease, and continuously moistened with warmed $1 \times$ PBS $\left(36^{\circ} \mathrm{C}\right)$. Mice hydration was maintained by intraperitoneal injection of $200 \mu \mathrm{l}$ $0.9 \%$ saline solution every hour. During the course of the experiment, mouse body temperature was maintained at $37^{\circ} \mathrm{C}$ using a heating pad, and oxygen saturation and heart rate were monitored using the pulse oximetry Physiosuit apparatus (Kent Scientific). The tail vein was cannulated allowing the injection of fluorescent dyes and/or bacteria.

Spinning-disk confocal microscope. Intravital imaging was performed using a Leica DM6 FS upright microscope equipped with a motorized stage. The microscope is fitted with HCX PL Fluotar 5x/0.15, HC Fluotar $\times 25 / 0.95$ and HC PL APO $\times 40$ / 1.10 objectives lens (Leica), mounted on an optical table to minimize vibration and totally enclosed in a biosafety cabinet (Noroit). The microscope is coupled to a Yokogawa CSU-W1 confocal head modified with Borealis technology (Andor). Four laser excitation wavelengths $(488,561,642$, and $730 \mathrm{~nm})$ were used in fast succession and visualized with the appropriate long-pass filters. Fluorescence signals were detected using a sCMOS $2048 \times 2048$ pixel camera (Orca Flash v2 +, Hamamatsu). Metamorph acquisition software (Molecular devices) was used to drive the confocal microscope.

Imaging heterotypic interactions between murine neutrophils and human endothelium. Two hours prior to the skin flap surgery, mice were subcutaneously injected with $0.5 \mu \mathrm{g}$ of human recombinant TNFa (Preprotech, \#300-01A) under the human skin graft. After the skin flap surgery of grafted SCID/beige mice, the murine vasculature was labeled with $15 \mu$ g Dylight 488 -conjugated anti-mouse CD31 (LEAF purified anti-mouse CD31, clone 390, Biolegend, \#102412+ DyLight488 Antibody Labelling Kit, ThermoFisher Scientific, \#53024) and the human vasculature with $100 \mu \mathrm{g}$ of Dylight755-conjugated UEA-1 lectin (unconjugated UEA-1 lectin, Vector Laboratories, \#L-1060 + DyLight755 Antibody Labelling Kit, Thermofisher Scientific, \#84538). Neutrophils were labeled with 2.5 $\mu \mathrm{g}$ Dylight550-conjugated anti-mouse Ly-6G (LEAF purified anti-mouse Ly-6G, clone 1A8, Biolegend, \#127620 + DyLight550 Antibody Labelling Kit, ThermoFisher Scientific, \#84530) 15 min prior to recordings. Time-lapse z-stack series (1.5-2 $\mu \mathrm{m}$ spacing) were recorded every $30 \mathrm{~s}$ for $30 \mathrm{~min}$ at 3 and $4 \mathrm{~h}$ post TNFa stimulation. 
Imaging the early phase of infection (0-6h). After the skin flap surgery of grafted SCID/beige mice and $15 \mathrm{~min}$ prior to the tail-vein injection of iRFP-expressing bacteria ( $10^{7} \mathrm{CFU}$ in $\left.100 \mu \mathrm{l} 1 \times \mathrm{PBS}\right), 100 \mu \mathrm{g}$ Dylight755-conjugated UEA-1 lectin (unconjugated UEA-1 lectin, Vector Laboratories, \#L-1060 + DyLight755 Antibody Labelling Kit, Thermofisher Scientific, \#84538), $15 \mu \mathrm{g}$ Dylight488-conjugated anti-mouse CD31 (LEAF purified anti-mouse CD31, clone 390, Biolegend, \#102412 + DyLight488 Antibody Labelling Kit, ThermoFisher Scientific, \#53024) and $2.5 \mu \mathrm{g}$ Dylight550-conjugated anti-mouse Ly-6G (LEAF purified anti-mouse Ly-6G, clone 1A8, Biolegend, \#127620 + DyLight550 Antibody Labelling Kit, ThermoFisher Scientific, \#84530) were injected to label and visualize the human vessels, the mouse dermal microvasculature and the mouse neutrophils, respectively. $\mathrm{z}$-stack images (1.5-2 $\mu \mathrm{m}$ spacing) were captured every 15 or $30 \mathrm{~min}$ for 4 to $6 \mathrm{~h}$.

Imaging adhesion of bacteria in arterioles during the early phase of infection (0-3h). Two hours prior to the skin flap surgery, mice were intravenously injected with Hydrazide AlexaFluor633 (2 mg kg-1, ThermoFisher Scientific, \#30633) to label arteriole walls, as previously described ${ }^{21}$. After the skin flap surgery and $15 \mathrm{~min}$ prior to the tail-vein injection of GFP-expressing bacteria $\left(10^{7} \mathrm{CFU}\right.$ in $100 \mu \mathrm{l} 1 \times$ PBS), $100 \mu \mathrm{g}$ Dylight755-conjugated UEA-1 lectin (unconjugated UEA-1 lectin, Vector Laboratories, \#L-1060 + DyLight755 Antibody Labelling Kit, Thermofisher Scientific, \#84538) were injected in the mouse tail vein to label and visualize the human vessels. $\mathrm{z}$-stack images (1.5-2 $\mu \mathrm{m}$ spacing) were captured every $30 \mathrm{~min}$ for $3 \mathrm{~h}$.

Imaging the late phase of infection ( $>16 \mathrm{~h}$ ). In all, $16 \mathrm{~h}$ prior imaging, grafted $\mathrm{Rag}_{2}{ }^{-1}$ ${ }^{-} \gamma_{c}{ }^{-1-} L y s M g f p /++$ mice were retro-orbitally injected with iRFP-expressing bacteria $\left(10^{7} \mathrm{CFU}\right.$ in $\left.100 \mu \mathrm{l} 1 \times \mathrm{PBS}\right)$. The skin flap surgery was initiated $15 \mathrm{~h}$ post infection and time-lapse z-stack series (1.5-2 $\mu \mathrm{m}$ spacing) of iRFP-expressing bacteria and eGFP-expressing neutrophils were recorded every $30 \mathrm{~s}$ for $30 \mathrm{~min}$.

Imaging human E-selectin (CD62E) expression during the early phase of infection. After the skin flap surgery and 15 min prior to the tail-vein injection of GFPexpressing bacteria ( $10^{7} \mathrm{CFU}$ in $\left.100 \mu \mathrm{l} 1 \times \mathrm{PBS}\right)$ and PE-conjugated anti-human CD62E (clone P2H3, $6 \mu \mathrm{g}$, Thermofisher Scientific, \#12-0627-42), $100 \mu \mathrm{g}$ Dylight755-conjugated UEA-1 lectin (unconjugated UEA-1 lectin, Vector Laboratories, \#L-1060 + DyLight755 Antibody Labelling Kit, Thermofisher Scientific, \#84538), and $50 \mu \mathrm{g}$ of Hydrazide AlexaFluor633 were injected in the mouse tail vein to label and visualize the human vessels and the arteriole walls, respectively. $z$-stack images ( $2 \mu \mathrm{m}$ spacing) were captured every $60 \mathrm{~min}$ for $4 \mathrm{~h}$. To block the function of human E-selectin, $30 \mu \mathrm{g}$ of anti-E-selectin blocking antibody (clone P2H3, Thermofisher Scientific, \#14-0627-82) were intravenously injected $5 \mathrm{~min}$ prior to mouse infection. Control experiments were performed following the intravenous injection of $30 \mu \mathrm{g}$ of the mouse IgG1к isotype control (clone P3.6.2.8.1, Thermofisher Scientific, \#14-4714-85).

Image processing. The images were exported from the Metamorph acquisition software (Molecular Devices) as .tiff files, deconvoluted using Huygens software (Scientific Volume Imaging), and edited using Fiji. Movies were exported as avi files and edited on Final Cut Pro (Apple). 3D-rendering was generated using Imaris software (Bitplane).

In vivo neutrophil depletion. Neutrophils were depleted in grafted mice by intravenous injection of either $50 \mu \mathrm{g}$ of anti-mouse Ly-6G/Ly-6C (GR-1) monoclonal antibody clone RB6-8C5 (eBioscience, \#16-5931-85) or $100 \mu \mathrm{g}$ of anti-mouse Ly-6G monoclonal antibody clone 1A8 (eBioscience, \#16-9668-85) $24 \mathrm{~h}$ prior to infection. Control animals were injected with the respective rat IgG2 $\beta, \mathrm{K}$ or eBrD isotype control (eBioscience, \#16-4031-85 and \#14-4321-85, respectively). Neutrophil depletion was confirmed by differential leukocyte count and flow cytometry analysis.

Evaluation of vascular permeability with Evans blue. Twenty-four hours post infection, anesthetized mice received an intravenous injection of $100 \mu \mathrm{l}$ sterile Evans blue dye $(0.5 \% \mathrm{w} / \mathrm{v}$ in $1 \times \mathrm{PBS})$ that was allowed to circulate for $10 \mathrm{~min}$. Mice were then perfused with $5 \mathrm{ml}$ of heparin-containing $1 \times \mathrm{PBS}\left(2 \mathrm{units}^{-1}\right)$ and 4 $\mathrm{mm}^{2}$ biopsies of the human xenografts were harvested using dermatological biopsy punchers. Skin biopsies were weighed, disposed into $1.5-\mathrm{ml}$ tubes containing $300 \mu \mathrm{l}$ formamide, and homogenized using MagNA lyser homogenizer set at $6000 \times g$ for $30 \mathrm{sec}$. Samples were incubated at $37^{\circ} \mathrm{C}$ for $60 \mathrm{~h}$ to extract Evans blue dye from the tissues and centrifuged to pellet any remaining tissue fragments. The optical densities at $620 \mathrm{~nm}$ of the supernatants were measured using a spectrophotometer and the Evans blue concentration $\left(\mathrm{ng} \mathrm{ml}^{-1}\right)$ of each sample was determined.

Quantification of vascular damage. Images of H\&E-stained skin sections were manually analyzed and more than 200 blood dermal vessels were assessed for every condition at indicated times and classified as healthy, congested (i.e., luminal accumulation of red blood cells), or breached (i.e., presence of perivascular red blood cells) vessels. The percentage of vessels in each condition was then determined.

Statistics. All graphs and statistical analyses were performed with GraphPad Prism 8 (GraphPad Software). No statistical method was used to predetermine sample size. Kolmogorov-Smirnov test was used to assess the normality of all data sets. Scatter dot plots show the mean \pm SEM. $P$ values were considered as statistically significant when inferior at 0.05 . Statistical details of experiments (sample size, replicate number, statistical significance) can be found in the figures, figure legends, and source data files.

Reporting summary. Further information on research design is available in the Nature Research Reporting Summary linked to this article.

\section{Data availability}

Data supporting this work are available in the main figures and the Supplementary Information files. Source data are provided with this paper.

Received: 3 May 2021; Accepted: 30 June 2021; Published online: 27 July 2021

\section{References}

1. Brandtzaeg, P. \& van Deuren, M. Classification and pathogenesis of meningococcal infections. Methods Mol. Biol. 799, 21-35 (2012).

2. van de Beek, D., de Gans, J., Tunkel, A. R. \& Wijdicks, E. F. Communityacquired bacterial meningitis in adults. N. Engl. J. Med. 354, 44-53 (2006).

3. Stephens, D. S. Conquering the meningococcus. FEMS Microbiol. Rev. 31, 3-14 (2007).

4. van Deuren, M., Brandtzaeg, P. \& van der Meer, J. W. Update on meningococcal disease with emphasis on pathogenesis and clinical management. Clin. Microbiol. Rev. 13, 144-166 (2000).

5. Harrison, O. B. et al. Analysis of pathogen-host cell interactions in purpura fulminans: expression of capsule, type IV pili, and PorA by Neisseria meningitidis in vivo. Infect. Immun. 70, 5193-5201 (2002).

6. Sotto, M. N., Langer, B., Hoshino-Shimizu, S. \& de Brito, T. Pathogenesis of cutaneous lesions in acute meningococcemia in humans: light, immunofluorescent, and electron microscopic studies of skin biopsy specimens. J. Infect. Dis. 133, 506-514 (1976).

7. Guarner, J. et al. Pathogenesis and diagnosis of human meningococcal disease using immunohistochemical and PCR assays. Am. J. Clin. Pathol. 122, 754-764 (2004).

8. Melican, K., Michea Veloso, P., Martin, T., Bruneval, P. \& Dumenil, G. Adhesion of Neisseria meningitidis to dermal vessels leads to local vascular damage and purpura in a humanized mouse model. PLoS Pathog. 9, el003139 (2013).

9. Obino, D. \& Dumenil, G. The many faces of bacterium-endothelium interactions during systemic infections. Microbiol. Spectr. 7, https://doi.org/ 10.1128/microbiolspec.BAI-0010-2019 (2019).

10. Lerolle, N. et al. Assessment of the interplay between blood and skin vascular abnormalities in adult purpura fulminans. Am. J. Respir. Crit. Care Med. 188, 684-692 (2013)

11. Li, H. et al. SARS-CoV-2 and viral sepsis: observations and hypotheses. Lancet 395, 1517-1520 (2020).

12. Bonazzi, D. et al. Intermittent pili-mediated forces fluidize Neisseria meningitidis aggregates promoting vascular colonization. Cell https://doi.org/ 10.1016/j.cell.2018.04.010 (2018)

13. Yan, H. C. et al. Human/severe combined immunodeficient mouse chimeras An experimental in vivo model system to study the regulation of human endothelial cell-leukocyte adhesion molecules. J. Clin. Investig. 91, 986-996 (1993).

14. Faust, N., Varas, F., Kelly, L. M., Heck, S. \& Graf, T. Insertion of enhanced green fluorescent protein into the lysozyme gene creates mice with green fluorescent granulocytes and macrophages. Blood 96, 719-726 (2000).

15. Carbonnelle, E., Helaine, S., Nassif, X. \& Pelicic, V. A systematic genetic analysis in Neisseria meningitidis defines the Pil proteins required for assembly, functionality, stabilization and export of type IV pili. Mol. Microbiol. 61, 1510-1522 (2006).

16. Nassif, X. et al. Antigenic variation of pilin regulates adhesion of Neisseria meningitidis to human epithelial cells. Mol. Microbiol 8, 719-725 (1993).

17. Finsterbusch, M., Voisin, M. B., Beyrau, M., Williams, T. J. \& Nourshargh, S. Neutrophils recruited by chemoattractants in vivo induce microvascular plasma protein leakage through secretion of TNF. J. Exp. Med. 211, 1307-1314 (2014).

18. Hou, S. T. et al. Semaphorin3A elevates vascular permeability and contributes to cerebral ischemia-induced brain damage. Sci. Rep. 5, 7890 (2015). 
19. Koehl, B. et al. The endothelin B receptor plays a crucial role in the adhesion of neutrophils to the endothelium in sickle cell disease. Haematologica 102, 1161-1172 (2017).

20. Ho, M., Hickey, M. J., Murray, A. G., Andonegui, G. \& Kubes, P. Visualization of Plasmodium falciparum-endothelium interactions in human microvasculature: mimicry of leukocyte recruitment. J. Exp. Med. 192, 1205-1211 (2000).

21. Shen, Z., Lu, Z., Chhatbar, P. Y., O’Herron, P. \& Kara, P. An artery-specific fluorescent dye for studying neurovascular coupling. Nat. Methods 9, 273-276 (2012).

22. Ley, K., Laudanna, C., Cybulsky, M. I. \& Nourshargh, S. Getting to the site of inflammation: the leukocyte adhesion cascade updated. Nat. Rev. Immunol. 7, 678-689 (2007).

23. Dixon, G. L., Heyderman, R. S., van der Ley, P. \& Klein, N. J. High-level endothelial E-selectin (CD62E) cell adhesion molecule expression by a lipopolysaccharide-deficient strain of Neisseria meningitidis despite poor activation of NF-kappaB transcription factor. Clin. Exp. Immunol. 135, 85-93 (2004).

24. Hickey, M. J. et al. Varying roles of E-selectin and P-selectin in different microvascular beds in response to antigen. J. Immunol. 162, 1137-1143 (1999).

25. Runnels, J. M. et al. Imaging molecular expression on vascular endothelial cells by in vivo immunofluorescence microscopy. Mol. Imaging 5, 31-40 (2006).

26. Yan, H. C. et al. Leukocyte recruitment into human skin transplanted onto severe combined immunodeficient mice induced by TNF-alpha is dependent on E-selectin. J. Immunol. 152, 3053-3063 (1994).

27. Milstone, D. S. et al. Mice lacking E-selectin show normal numbers of rolling leukocytes but reduced leukocyte stable arrest on cytokine-activated microvascular endothelium. Microcirculation 5, 153-171 (1998).

28. Mairey, E. et al. Cerebral microcirculation shear stress levels determine Neisseria meningitidis attachment sites along the blood-brain barrier. J. Exp. Med. 203, 1939-1950 (2006).

29. Mikaty, G. et al. Extracellular bacterial pathogen induces host cell surface reorganization to resist shear stress. PLoS Pathog. 5, e1000314 (2009).

30. Nourshargh, S., Renshaw, S. A. \& Imhof, B. A. Reverse migration of neutrophils: where, when, how, and why? Trends Immunol. 37, 273-286 (2016).

31. Sumagin, R. \& Sarelius, I. H. Emerging understanding of roles for arterioles in inflammation. Microcirculation 20, 679-692 (2013).

32. Sumagin, R. \& Sarelius, I. H. A role for ICAM-1 in maintenance of leukocyteendothelial cell rolling interactions in inflamed arterioles. Am. J. Physiol. Heart Circ. Physiol. 293, H2786-H2798 (2007).

33. Pastore, L. et al. Angiotensin II stimulates intercellular adhesion molecule-1 (ICAM-1) expression by human vascular endothelial cells and increases soluble ICAM-1 release in vivo. Circulation 100, 1646-1652 (1999).

34. Sollberger, G., Tilley, D. O. \& Zychlinsky, A. Neutrophil extracellular traps: the biology of chromatin externalization. Dev. Cell 44, 542-553 (2018).

35. Contou, D. et al. Clinical spectrum and short-term outcome of adult patients with purpura fulminans: a French multicenter retrospective cohort study. Intensive Care Med. 44, 1502-1511 (2018).

36. Rusniok, C. et al. NeMeSys: a biological resource for narrowing the gap between sequence and function in the human pathogen Neisseria meningitidis. Genome Biol. 10, R110 (2009).

37. Morand, P. C., Tattevin, P., Eugene, E., Beretti, J. L. \& Nassif, X. The adhesive property of the type IV pilus-associated component PilC1 of pathogenic Neisseria is supported by the conformational structure of the N-terminal part of the molecule. Mol. Microbiol. 40, 846-856 (2001).

38. Filonov, G. S. et al. Bright and stable near-infrared fluorescent protein for in vivo imaging. Nat. Biotechnol. 29, 757-761 (2011)

39. Baranska, A. et al. Unveiling skin macrophage dynamics explains both tattoo persistence and strenuous removal. J. Exp. Med. 215, 1115-1133 (2018).

40. Yu, Y. R. et al. A protocol for the comprehensive flow cytometric analysis of immune cells in normal and inflamed murine non-lymphoid tissues. PLoS ONE 11, e0150606 (2016).

41. Schindelin, J. et al. Fiji: an open-source platform for biological-image analysis. Nat. Methods 9, 676-682 (2012).

\section{Acknowledgements}

The authors thank Elisa Gomez Perdiguero, Molly Ingersoll, Ana-Maria LennonDuménil, and Benoît Marteyn for fruitful discussions and critical reading of the manuscript. We would like to thank Maxime Gauberti for his insights into the expression of human inflammatory adhesion receptors. We also thank Albane Imbert (FabLab Institut Pasteur (FLIP), DRTE, Institut Pasteur, Paris, France) for 3D-printing, Michael Hivelin (Hôpital Européen Georges Pompidou) for providing skin samples, Sophie Novault (Cytometry and Biomarkers UTechS, Institut Pasteur, Paris, France) for technical advice, Eric Camerer (UMR-970, Paris-Cardiovascular Research Center PARCC, Paris, France) for technical advice on vascular permeability experiments and the mem bers of the UtechS Photonic BioImaging (Imagopole, C2RT, Institut Pasteur, Paris, France), supported by the French National Research Agency (France BioImaging; ANR10-INSB-04; investments for the Future) for assistance in image acquisition and analysis. This work was supported by the Integrative Biology of Emerging Infectious Diseases (IBEID) laboratory of excellence (ANR-10-LABX-62), and the VIP European Research Council-starting grant (310790-VIP, G.D.). D.O. was supported by a Pasteur-Roux Postdoctoral Fellowship from the Institut Pasteur. H.E.-R. was supported by a Marie Curie Intra-European Fellowship within the 7th project European Community Framework Program (624740).

\section{Author contributions}

G.D., D.O. and V.M. designed the research; V.M., P.N., T.U., H.E.-R., K.M., M.-P.F.-G., P.F. and D.O. performed experiments; T.S. provided human skin samples; P.B. performed and interpreted the immunohistochemistry analysis of the human case; V.M., P.N. and D.O. analyzed the data; D.O. assembled figures; D.O. and V.M. participated in manuscript writing; and G.D. supervised overall data analysis and wrote the manuscript

\section{Competing interests}

The authors declare no competing interests.

\section{Additional information}

Supplementary information The online version contains supplementary material available at https://doi.org/10.1038/s41467-021-24797-z.

Correspondence and requests for materials should be addressed to D.O. or G.Dén.

Peer review information Nature Communications thanks Tim Lämmermann, Hank Seifert and the other, anonymous, reviewer for their contribution to the peer review of this work. Peer reviewer reports are available.

Reprints and permission information is available at http://www.nature.com/reprints

Publisher's note Springer Nature remains neutral with regard to jurisdictional claims in published maps and institutional affiliations.

Open Access This article is licensed under a Creative Common Attribution 4.0 International License, which permits use, sharing, adaptation, distribution and reproduction in any medium or format, as long as you give appropriate credit to the original author(s) and the source, provide a link to the Creative Commons license, and indicate if changes were made. The images or other third party material in this article are included in the article's Creative Commons license, unless indicated otherwise in a credit line to the material. If material is not included in the article's Creative Commons license and your intended use is not permitted by statutory regulation or exceeds the permitted use, you will need to obtain permission directly from the copyright holder. To view a copy of this license, visit http://creativecommons.org/ licenses/by/4.0/

(C) The Author(s) 2021 